\title{
Mesenchymal stromal cell exosomes prevent and revert experimental pulmonary fibrosis through modulation of monocyte phenotypes
}

\author{
Nahal Mansouri, ${ }^{1,2,3,4,5}$ Gareth R. Willis, ${ }^{1,2}$ Angeles Fernandez-Gonzalez, ${ }^{1,2}$ Monica Reis, ${ }^{1,2}$ \\ Sina Nassiri, ${ }^{4,6}$ S. Alex Mitsialis, ${ }^{1,2}$ and Stella Kourembanas ${ }^{1,2}$ \\ 'Division of Newborn Medicine, Department of Pediatrics, Boston Children's Hospital (BCH), Boston, Massachusetts, USA. \\ 2Department of Pediatrics, Harvard Medical School, Boston, Massachusetts, USA. ${ }^{3}$ Pulmonary and Critical Care Medicine, \\ Brigham and Women's Hospital, Boston, Massachusetts, USA. ${ }^{4}$ Swiss Institute for Experimental Cancer Research (ISREC), \\ School of Life Sciences, École Polytechnique Fédérale de Lausanne (EPFL), Lausanne, Switzerland. ${ }^{5}$ Division of Pulmonary \\ Medicine, Department of Medicine, Lausanne University Hospital (CHUV), University of Lausanne (UNIL), Lausanne, \\ Switzerland. ${ }^{6}$ Bioinformatics Core Facility, SIB Swiss Institute of Bioinformatics, Lausanne, Switzerland.
}

\begin{abstract}
Mesenchymal stromal/stem cell (MSC) therapy has shown promise in experimental models of idiopathic pulmonary fibrosis (IPF). The aim of this study was to test the therapeutic effects of extracellular vesicles produced by human BM MSCs (MEx) in a bleomycin-induced pulmonary fibrosis model and investigate mechanisms of action. Adult C57BL/6 mice were challenged with endotracheal instillation of bleomycin and treated with MEx concurrently, or for reversal models, at day 7 or 21. Experimental groups were assessed at day 7, 14, or 28. Bleomycin-challenged mice presented with severe septal thickening and prominent fibrosis, and this was effectively prevented or reversed by MEx treatment. MEx modulated lung macrophage phenotypes, shifting the proportions of lung proinflammatory/classical and nonclassical monocytes and alveolar macrophages toward the monocyte/macrophage profiles of control mice. A parallel immunomodulatory effect was demonstrated in the BM. Notably, transplantation of MExpreconditioned BM-derived monocytes alleviated core features of pulmonary fibrosis and lung inflammation. Proteomic analysis revealed that MEx therapy promotes an immunoregulatory, antiinflammatory monocyte phenotype. We conclude that MEx prevent and revert core features of bleomycin-induced pulmonary fibrosis and that the beneficial actions of MEx may be mediated via systemic modulation of monocyte phenotypes.
\end{abstract}

Conflict of interest: SAM and SK are inventors of intellectual property technology licensed by BCH to United Therapeutics Corp.

Copyright: () 2019, American Society for Clinical Investigation.

Submitted: February 11, 2019 Accepted: September 27, 2019 Published: October 3, 2019.

Reference information: /CI Insight. 2019;4(21):e128060.

https://doi.org/10.1172/jici. insight.128060.

\section{Introduction}

Idiopathic pulmonary fibrosis (IPF) is a chronic progressive respiratory disease that is characterized by clinical features such as shortness of breath, hypoxemia, radiographically evident pulmonary infiltrates, and continuing accumulation of fixed fibrosis (1-3). Arguably, the complex interplay between immune cell subsets, coupled with an incomplete understanding in disease pathophysiology, have contributed to the paucity of successful therapies (4-6). In turn, IPF remains a fatal disease with, at present, a 5-year survival rate of less than $10 \%$ from the time of diagnosis (7-9). Thus, with no effective therapy for either the prevention or treatment of IPF, the need for new therapies is paramount.

To this end, novel approaches are required to address this multifactorial progressive disease. Interestingly, in experimental models of pulmonary fibrosis, mesenchymal stromal/stem cell (MSC) therapy has shown promise, reducing lung collagen deposition, improving Ashcroft score, and decreasing inflammatory markers in bronchoalveolar lavage (10-14). Despite such physiological improvements in the recipient lung following MSC transplantation, there is a burgeoning awareness that the mechanism of therapeutic action is predominantly paracrine. Indeed, one of the major therapeutic modalities identified in the MSC secretome are extracellular vesicles (EVs), including exosomes, the EV subset that is generated through the endocytic/endosomal pathway (15-18). Recently, we and others have shown that i.v. delivery of purified 
human MSC-derived exosomes (MEx) has provided substantial functional and immunomodulatory benefits in several experimental models of lung disease $(15,19,20)$ characterized by low levels of fibrosis. In this study, we utilize the murine bleomycin-induced lung injury model to investigate the therapeutic and immunomodulatory capacity of MEx on IPF pathology, a disease with prominent features of fibrosis.

\section{Results}

Purification, isolation, and characterization of exosomes. Exosomes were isolated from fraction 9 (F9) of concentrated cell culture supernatants after flotation on an iodixanol cushion (Figure 1A). Transmission electron microscopy (TEM) and nanoparticle tracking analysis (NTA) revealed that both human BM MSCs and human dermal fibroblasts (HDFs) gave rise to a heterogeneous exosome population that occupied a diameter of $\sim 35-150 \mathrm{~nm}$ and exhibited the typical morphological features of exosomes (Figure 1, B and C). Purified MEx and fibroblast-derived exosome (FEx) fraction (F9) had comparable particle counts (8.6 \pm $1 \times 10^{10}$ and $9.5 \pm 1 \times 10^{10}$, respectively) (Figure 1A, data for FEx not shown). Immunoblots demonstrated that all exosome preparations were positive for established exosome markers (CD63, ALIX, Flotillin-1 [FLOT1], and CD9) and lacked the cellular marker GM130 (Figure 1D and Supplemental Figure 3; supplemental material available online with this article; https://doi.org/10.1172/jci.insight.128060DS1).

$A$ bolus dose of MEx prevents and reverts bleomycin-induced pulmonary fibrosis. We used the murine bleomycin-induced experimental model of pulmonary fibrosis to assess the therapeutic capacity of MEx (21). First, to evaluate the preventive effect of MEx, 14-week-old mice received a single dose (endotracheal administration) of bleomycin (3 $\mathrm{U} / \mathrm{kg}$ ) or saline (vehicle control) on day 0 . Mice that received bleomycin were compared with an untreated control group. Concurrent with bleomycin administration (day 0 ), treatment groups received a single i.v. dose of MEx, FEx, or exosome-free iodixanol vehicle. Mice were sacrificed at day 14, and lung sections were assessed for quantification of fibrosis (Masson's trichrome) and collagen content (schematic shown in Figure 2A). Bleomycin-exposed mice demonstrated a histological pattern akin to human IPF, characterized by severe septal thickening and prominent fibrosis (Figure $2 \mathrm{~B}$ ). Accordingly, compared with the control group, animals that received bleomycin presented with a greater Ashcroft score (1.76 \pm 0.6 vs. $7.2 \pm 0.3, P<0.001$, Figure $2 \mathrm{C}$ ), coupled with an elevated degree of collagen deposition ( $2.18 \pm 0.09$ vs. $3.02 \pm 0.23 \mathrm{mg} / \mathrm{mL}, P<0.05$, Figure $2 \mathrm{D})$. A single i.v. dose of MEx dramatically blunted the bleomycin-induced pulmonary fibrosis, improved the Ashcroft score $(2.7 \pm 0.6, P<0.0001)$, and restored collagen content to levels similar to their untreated-counterparts $(2.18 \pm 0.15 \mathrm{mg} / \mathrm{mL}, P<$ 0.05). FEx and exosome-free iodixanol served as biologic and treated-vehicle controls, respectively, and had no effect on pulmonary fibrosis, Ashcroft score, or collagen deposition $(P>0.05$, Figure 2, B-D).

Given the critical role of apoptosis in the pathogenesis of fibrotic lung diseases $(22,23)$, we also investigated the antiapoptotic effect of MEx in the lung parenchyma. Here, both flow cytometric analysis of annexin V and TUNEL staining in whole lung sections revealed that bleomycin control mice presented with elevated levels of whole lung apoptosis compared with untreated control animals and that MEx treatment efficiently reduced the degree of apoptosis (Figure 2, E-G).

In addition to the preventive capacity of MEx, we also assessed the ability of MEx to revert bleomycin-induced pulmonary fibrosis after the injury occurred. Here, following endotracheal bleomycin instillation at day 0 , we administered a bolus dose of MEx at day 7 and assessed experimental groups at day 14 (schematic shown in Figure 3A). Notably, a single MEx dose substantially reverted bleomycin-induced pulmonary fibrosis, improved the Ashcroft score $(P<0.0001)$, and restored collagen content to levels akin to their untreated counterparts $(P<0.05$, Figure 3, B-D).

We also sought to test the capacity of MEx to revert core features of bleomycin-induced pulmonary fibrosis at a late rescue time point. Here, mice that received bleomycin (day 0) were given a bolus MEx dose at day 21 and assessed at day 28 (schematic shown in Figure 3E). Compared with bleomycin control animals, late-rescue MEx treatment significantly reduced the bleomycin-induced elevation in collagen content $(P<0.0001$, Figure $3, \mathrm{~F}$ and $\mathrm{G})$, although no difference was noted in the Ashcroft score $(P>0.05$, Figure $3, \mathrm{~F}$ and $\mathrm{H})$.

MEx treatment modulates alveolar macrophage and monocyte populations in the lung. Alveolar macrophages (alveolar MФ) and infiltrating monocytes play a pivotal role in pulmonary inflammation and in the development and progression of fibrosis (24-26). To investigate changes in immune cell populations following bleomycin-induced lung injury, we performed whole lung cytometric analysis at days 7 and 14 in animals that received MEx at day 0 . On day 7, compared with control mice, we noted a decrease in the proportion of alveolar MФ and nonclassical monocytes (defined as $\mathrm{CD} 45^{+} \mathrm{CD} 11 \mathrm{~b}^{-} \mathrm{CD} 11 \mathrm{c}^{+} \mathrm{CD} 64^{+}$cells and $\mathrm{CD} 45^{+}$ 


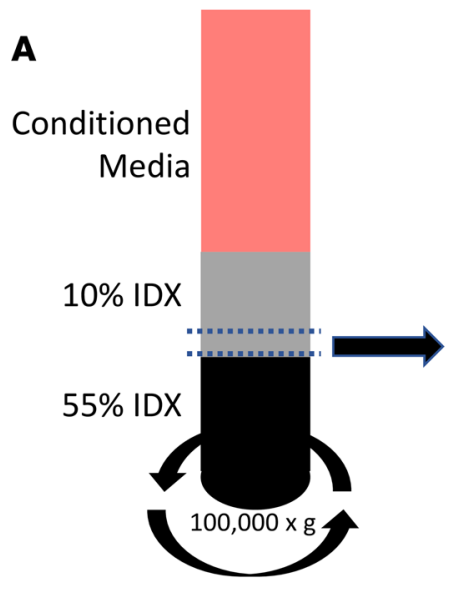

C

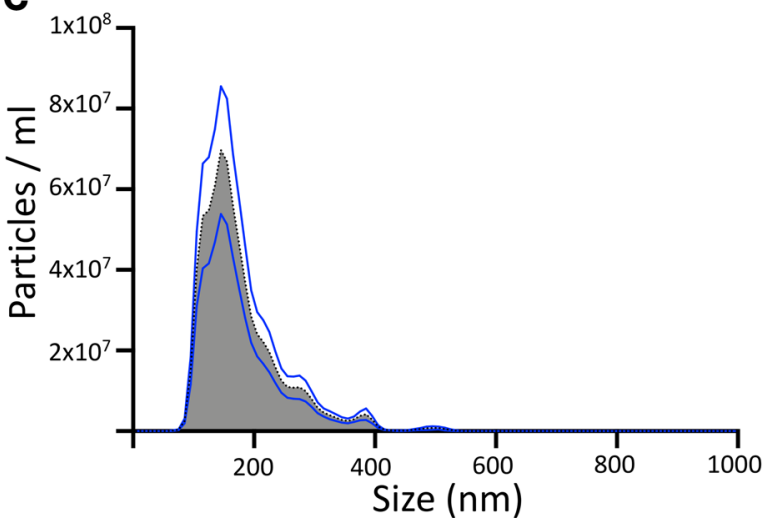

Purified MEx Fraction (F9)

\begin{tabular}{l|c}
\hline Density & $\sim 1.16-1.18 \mathrm{~g} / \mathrm{ml}$ \\
\hline Particle count & $8.6 \pm 1 \times 10^{10} / \mathrm{ml}$ \\
\hline Protein & $3 \pm 0.3 \mu \mathrm{g}$ \\
\hline Protein : Particle & $3 \pm 0.3 \times 10^{10} / \mathrm{ml}$ \\
\hline
\end{tabular}

B

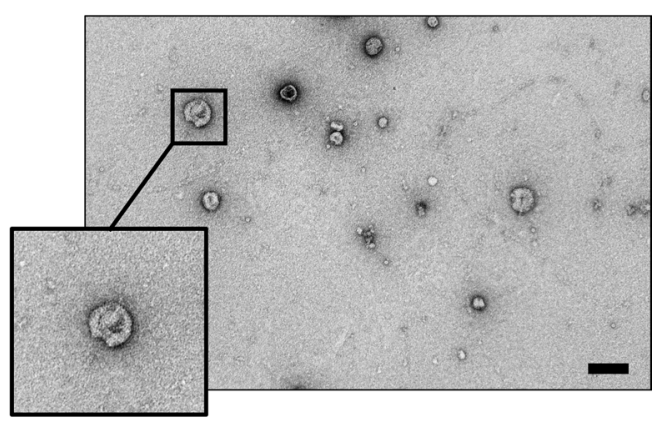

D

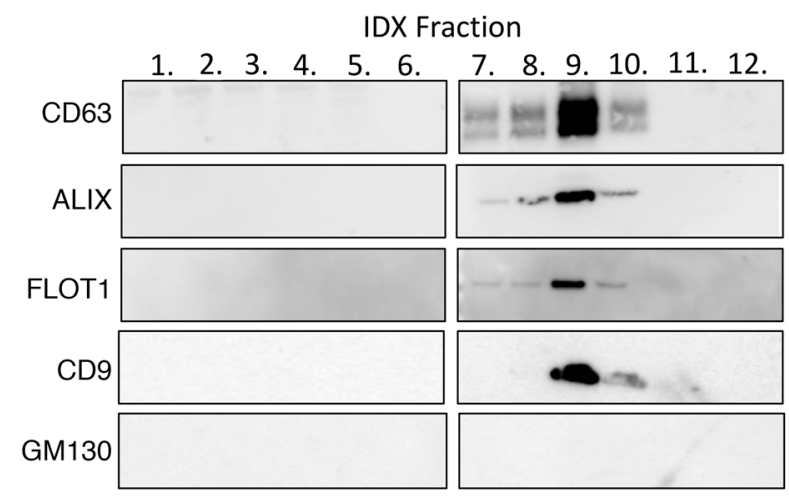

Figure 1. Exosome isolation, purification, and characterization. (A) Concentrated conditioned media (CM) was floated on an iodixanol (IDX) cushion gradient, and the purify exosome fraction was isolated from fraction 9 (F9; mesenchymal stromal/stem cell-extracellular vesicles/exosomes [MEx] density $\sim 1.16-1.18 \mathrm{~g} / \mathrm{mL}$ ). Nanoparticle tracking analysis (NTA) and protein concentration was used to assess exosome concentration and particle/protein ratio in the IDX cushion ( $12 \times 1 \mathrm{~mL}$ fractions), respectively. (B) Transmission electron microscopy images demonstrating heterogeneous vesicle morphology (scale bar: $500 \mathrm{~nm}$ ) . (C) Size distribution and particle concentration was measured by NTA. (D) The IDX cushion gradient fractions were analyzed by Western blot (fractions 1-6 and 7-12, side by side), using antibodies to proteins representing exosome markers. Equivalent volume of each fraction was loaded per lane. Representative images are shown. Flotillin 1 (FLOT1), ALIX, and tetraspanins (CD63, CD9) were enriched in F9. GM130 (cytoplasmic marker) was absent in F9.

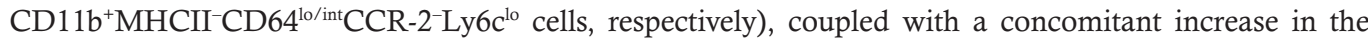
number of proinflammatory classical monocytes (defined as $\mathrm{CD} 45^{+} \mathrm{CD} 11 \mathrm{~b}^{+} \mathrm{MHCII}{ }^{-} \mathrm{CD} 64^{10 / \text { int }} \mathrm{CCR}-2^{+} \mathrm{Ly}-$ $6 c^{\text {hi }}$ cells) in bleomycin-exposed animals. MEx therapy effectively rescued all cell populations, increasing the levels of alveolar MФ $(P<0.01)$ and nonclassical monocytes $(P<0.05)$, while decreasing the number of classical monocytes comparable with controls $(P<0.001$, Figure $4 \mathrm{~A})$.

Paradoxically, at day 14 (Figure 4B), we found that the proportion of alveolar MФ was increased $(P<$ $0.05)$, while the number of classical monocytes were reduced in bleomycin-treated animals, compared with controls. Again, MEx therapy shifted the alveolar МФ and monocyte profiles toward that of their untreated counterparts. Importantly, cytometric analysis showed that the overall percentage of CD45 ${ }^{+}$monocytes did not change across the 3 experimental groups at either time point (day 7 and day 14). Representative gating strategy is shown in Figure 4C.

Administration of MEx modulates whole lung inflammation. To investigate the effect of MEx on pulmonary inflammation, whole lung mRNA levels were assessed at days 7 and 14 . We noted that gene expression levels of proinflammatory cytokines that were typically associated with activation of $M \Phi$, such as $C c l 2$ and arginase 1 ( $\operatorname{Arg} 1)$, were dramatically elevated in mice that received bleomycin compared with control animals at both 7 and 14 days), and that this bleomycin-induced elevation was reduced by MEx administration ( $P<0.05$, Figure $5, \mathrm{~A}$ and B). Il6 showed a similar trend, but the difference did not reach statistical significance between groups $(P>0.05)$. Tgfb expression was similar at day 7 and day 14 between all experimental groups $(P>0.05$; Figure $5, \mathrm{~A}$ and $\mathrm{B})$. In accordance, immunofluorescence analysis of lung tissue sections showed a marked increase in the expression of CD206 and 




Bleomycin

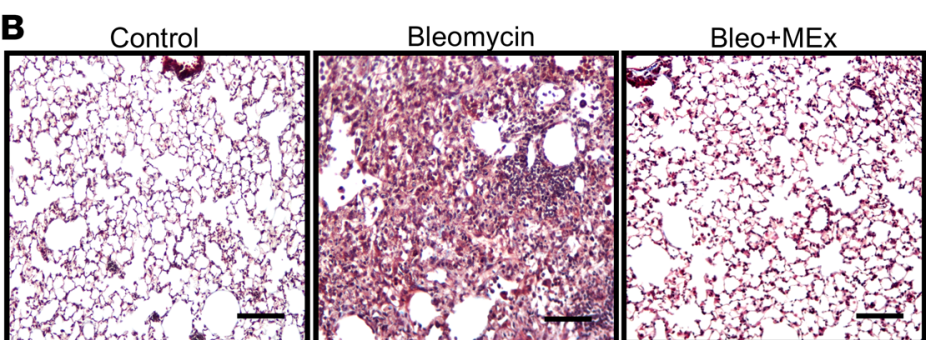

Control+MEX

Bleo+FEx

Bleo+IDX
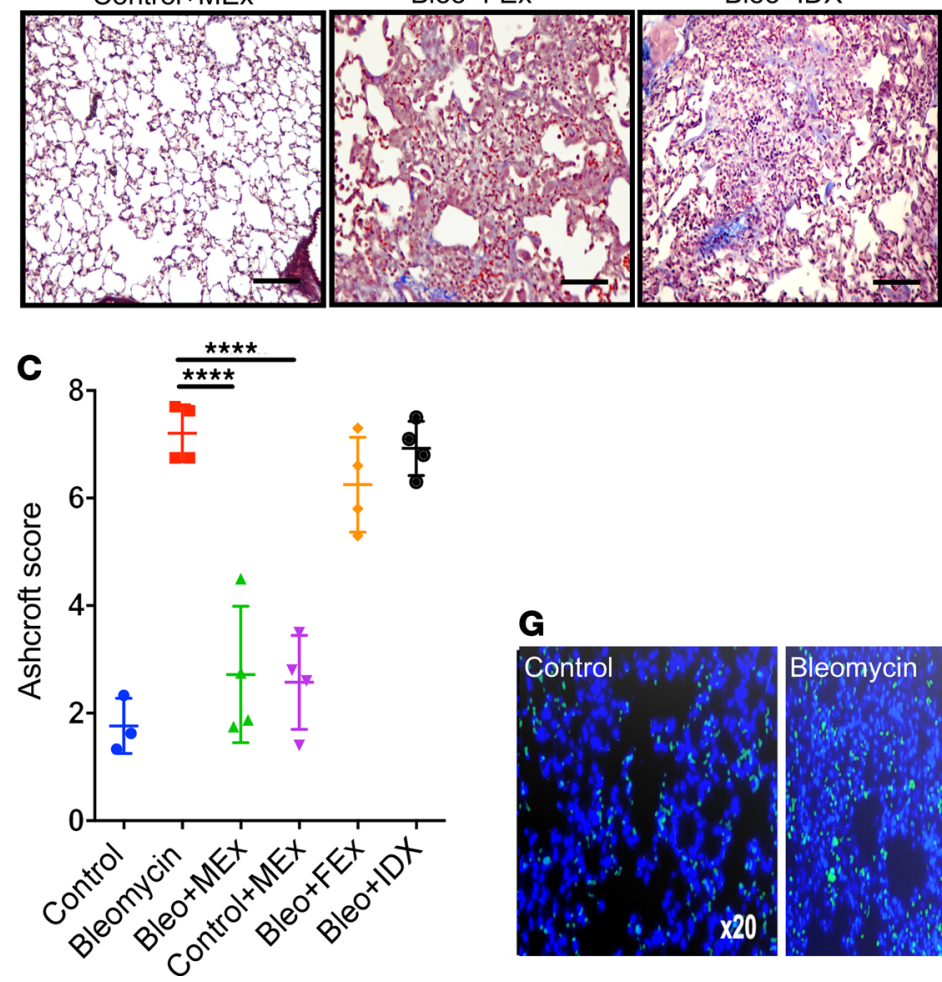

G
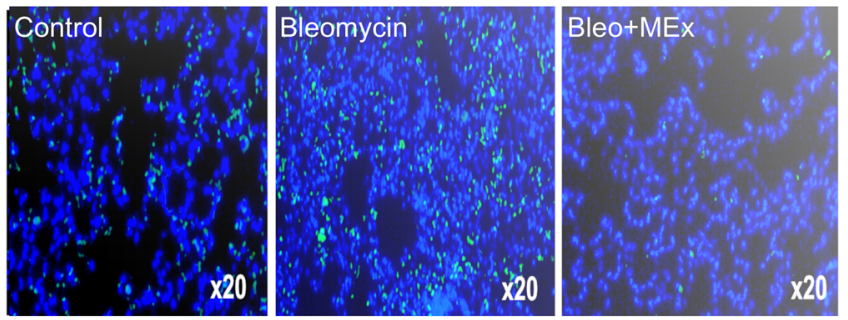

Figure 2. A bolus dose of MEx prevents bleomycin-induced pulmonary fibrosis. (A) Fourteen-week-old mice (C57BL/6 strain) received endotracheal bleomycin $(3 \mathrm{U} / \mathrm{kg}$ ) or $0.9 \%$ normal saline on day 0 (control). Concurrently, treated groups received a bolus i.v. dose of MEx (Bleo+MEx), FEx (Bleo+FEx), or iodixanol (Bleo+IDX). Mice were sacrificed on day 14. The cross symbol represents animal harvest. (B) Lung sections were stained with Masson's trichrome. Images were taken at ×100 magnification. Bleomycin, Bleo+FEx, and Bleo+IDX showed architectural destruction, alveolar septal thickening, and fibrotic changes. Administration of MEx to bleomycin-challenged mice substantially reduced fibrosis and alveolar distortion. Scale bar: $100 \mu \mathrm{m}$. (C) Lung fibrosis was measured at day 14 by Ashcroft score. (D) Collagen deposition was assessed by Sircol assay and represented as $\mathrm{mg} / \mathrm{mL}$ of left lung homogenate. (E) Data are representative of 3 independent experiments, mean \pm SD. $n=3-4$ per experimental group; each symbol represents 1 mouse. ${ }^{*} P<0.05$; ${ }^{* * *} P<0.0001$, 1-way ANOVA followed by Fisher's LSD post hoc analysis. (F and $\left.\mathbf{G}\right)$ MEx therapy decreases apoptosis. Annexin V/PI staining in whole lungs shows an increase in apoptosis (annexin $\mathrm{V}^{+} \mathrm{Pl}^{-}$) in bleomycin-exposed mice compared with control and bleomycin+MEx mice. TUNEL staining in whole lung sections shows increase in apoptosis (green) in the bleomycin-exposed group of mice compared with control and bleomycin+MEx. Nuclei were stained with DAPI. Images obtained at $\times 20$ magnification. MFI quantified using ImageJ software and normalized for DAPI. Data are representative of 2 independent experiments, mean \pm SD. $n=6-8$ per group; each symbol represents 1 mouse. ${ }^{*} P<0.05 ;{ }^{*} P<0.01$ vs. bleomycin-exposed mice. One-way ANOVA followed by Fisher's LSD post hoc analysis. MEx, mesenchymal stromal/stem cell-extracellular vesicles/exosomes; FEx, Human dermal fibroblast exosomes; IDX, iodixanol; Bleo, bleomycin; PI, propidium iodide. 
A

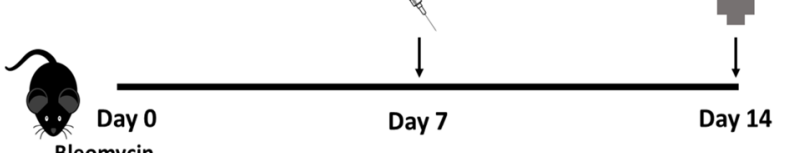

B
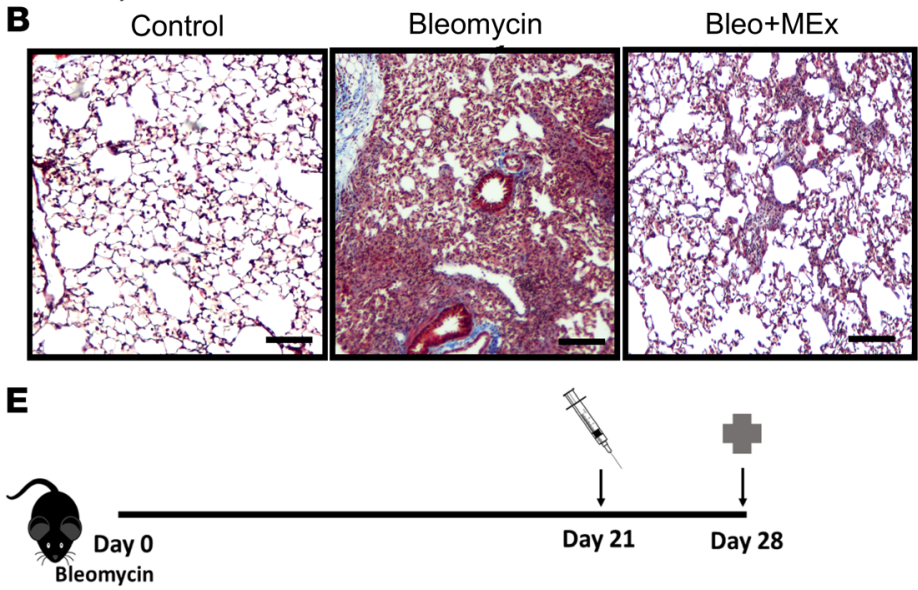

$\mathbf{F}$

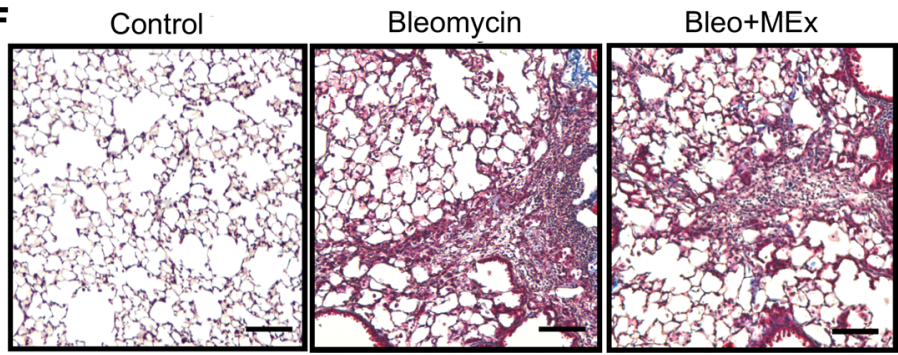

C

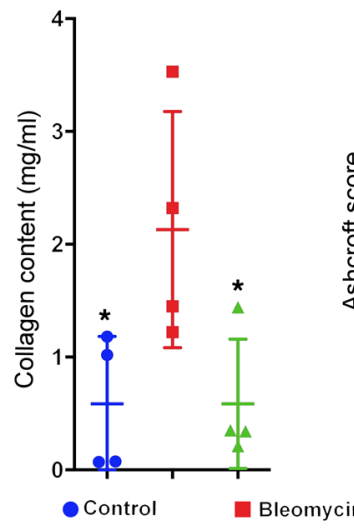

G

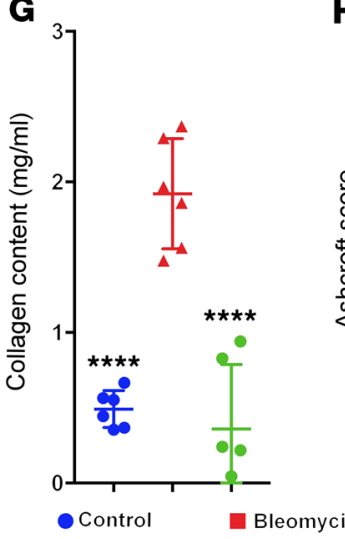

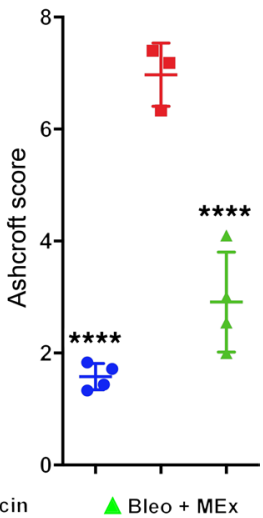

D

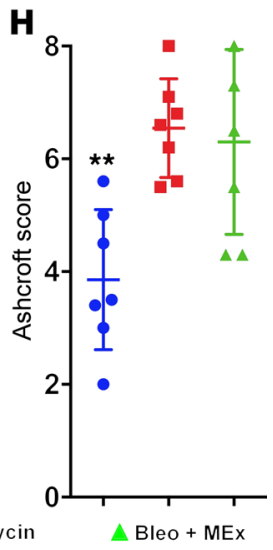

Figure 3. MEx therapy reverts bleomycin-induced pulmonary fibrosis. (A and E) MEx were administered 7 days or 21 days after the administration of bleomycin, and mice were sacrificed on day 14 or 28. Cross symbol represents animal harvest. (B and $\mathbf{F}$ ) Lung sections were stained with Masson's trichrome. Images were taken at $\times 100$ magnification. Scale bar: $100 \mu \mathrm{m}$. Representative lung sections from control, Bleomycin, and Bleo+MEx mice. (C, D, G, and $\mathbf{H}$ ) Lung sections were assessed for collagen deposition ( $\mathbf{C}$ and $\mathbf{G}$ ) and histology ( $\mathbf{D}$ and $\mathbf{H})$. Data represent mean \pm SD. Data in $\mathbf{A}-\mathbf{D}$ are representative of 3 independent experiments, $n=3-4$ per experimental group. Data in E-G are representative of 2 independent experiments, $n=5-6$ per experimental group; each symbol represents 1 mouse. ${ }^{*} P<0.05 ;{ }^{* *} P<0.01$; ${ }^{* * *} P<0.0001$. One-way ANOVA followed by Fisher's LSD post hoc analysis.

ARG1 proteins in bleomycin-exposed mice, and it was effectively reduced by MEx therapy to levels akin to the control group ( $P<0.01$; Figure $5, \mathrm{C}$ and $\mathrm{D})$. As expected, bronchoalveolar lavage (BAL) protein content was increased in bleomycin-exposed mice as opposed to control. MEx therapy decreased the BAL total protein content $(P<0.05$, Figure 5E).

MEx therapy modulates monocyte population in the BM. Recruited monocytes from the BM to the lung have been associated with the pathophysiology of pulmonary fibrosis (24-27) and considering our finding that MEx therapy was associated with modulation of monocyte populations in the lung, we next investigated if i.v.-delivered MEx exert immunomodulatory actions directly on BM progenitors. Here, mice were sacrificed at day 7 and myeloid cells in the BM were assessed by flow cytometry.

Interestingly, bleomycin-exposed mice presented with dramatically reduced levels of nonclassical monocytes present in the BM compared with control animals $(14.18 \% \pm 2.7$ vs $32.3 \% \pm 5.5, P<0.05$, respectively). A single dose of MEx therapy administered concomitantly with bleomycin, shifted the BM nonclassical monocyte profile $(27.57 \% \pm 5.7, P<0.05)$ toward that of their untreated counterparts. Moreover, the classical monocyte population in the bleomycin-exposed group was greater $(67.8 \% \pm 1.7)$ than the control group $(50.1 \% \pm 3.2, P<0.001)$, and effectively suppressed by MEx therapy $(57.5 \% \pm 3.9, P<0.05$, Figure 6A). Notably, cytometric analysis showed that the proportion of total monocytes did not change across the 3 experimental groups (Figure 6).

MEx therapy reprograms monocytes to a nonclassical phenotype. Since MEx therapy was associated with a "prohomeostatic" shift in lung МФ/monocyte phenotype and a concomitant rescued BM nonclassical 

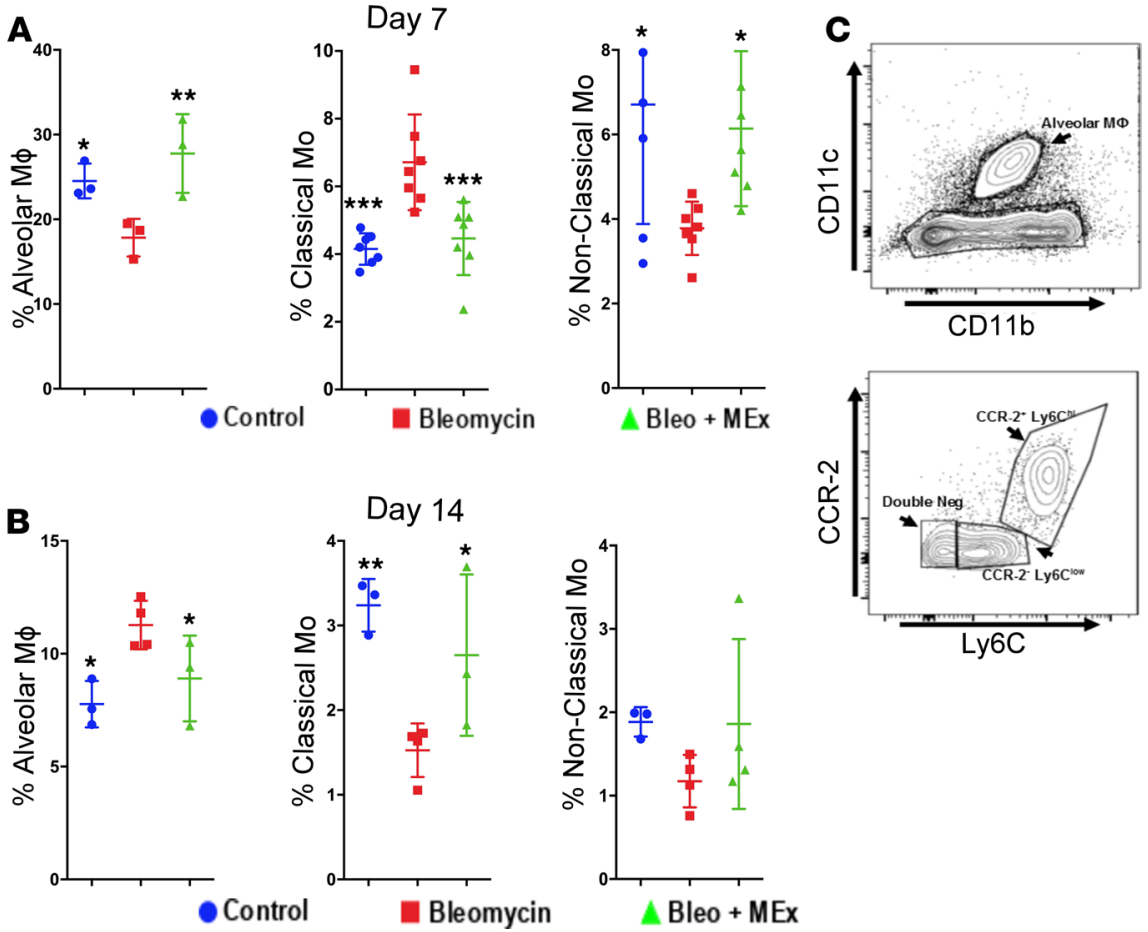

Figure 4. MEx therapy modulates alveolar macrophage and monocyte populations in the lung. (A and B) Flow cytometry was used to assess whole lung monocyte and alveolar macrophage (alveolar M $\Phi$ ) at day 7 (A) and day 14 (B). (C) Classical monocytes (Mo) were defined as CD45+CD11b+MHCII-CD64-CCR-2+Ly6C hi. Nonclassical monocytes were

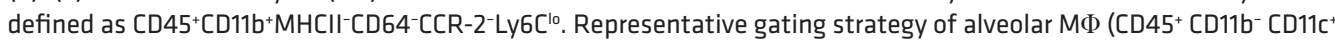
CD64+ cells), classical Mo, and nonclassical Mo. The gating strategy was performed according to fluorescence minus one controls (Supplemental Figure 1). Data are representative of 3 independent experiments, mean \pm SD, $n=4-5$ per experimental group; each symbol represents 1 mouse. ${ }^{*} P<0.05$; ${ }^{* *} P<0.01 ;{ }^{* *} P<0.001$. One-way ANOVA followed by Fisher's LSD post hoc analysis.

monocyte population, we sought to determine whether MEx therapy affords such robust physiological changes in the lung by directly modulating the BM-myeloid/monocyte (My/Mo) cell lineage phenotype. To investigate the modulatory effect of MEx on My/Mo populations, we preconditioned these cells with MEx ex vivo. Here, primary myeloid cells were isolated from the BM of WT (healthy) FVB mice aged 6-8 weeks. At day 0 of in vitro culture, the population of myeloid cells was heterogeneous with monocyte lineage accounting for approximately $24 \%$ of the cells in culture (BM-derived myeloid cells [BMDMy], data not shown). Cells were subsequently cultured for 3 days in the presence of MФ colony stimulating factor (M-CSF) to drive the monocyte/MФ lineage (28). Myeloid cells were treated with MEx or cell culture (MEx-free) medium alone (schematic shown in Figure 7A) and confirmed to be $\mathrm{CD} 45^{+}$and $\mathrm{CD} 11 \mathrm{~b}^{+}$by flow cytometry $(>90 \%$, Figure $7 \mathrm{~B})$. There was no difference in the number of viable cells and the degree of apoptosis between MEx treated or media (control) treated myeloid cells (data not shown). To understand the impact of MEx on My/Mo cells, we next profiled this cell lineage by performing proteome analysis after MEx treatment using Liquid Chromatography Tandem Mass Spectrometry (LC-MS/MS). To this end, we treated the My/Mo cells on day 1 and 2 with MEx (Figure 7A) or FEx (control) and harvested cells at day 3 for protein extraction. Analysis of the proteomic data revealed a signature composed of 84 features whose abundance varied significantly between MEx-treated My/ Mo cells and FEx-treated controls (FDR $<0.25$, Figure 7C). Interestingly, MEx treatment was associated with lower abundance of multiple proinflammatory proteins such as those belonging to the MAPK and the serpin family. On the other hand, MEx increased the abundance of proregulatory proteins such as $\mathrm{Mrc1}$ and CerS2. We next set out to test if this proteomic signature was indicative of either a classical or a nonclassical phenotype in MEx-treated monocytes using publicly available transcriptome data of Ly6 $\mathrm{c}^{\mathrm{pos}}$ and Ly6 $\mathrm{c}^{\text {neg }}$ BM-derived monocytes (BMDMo) (29). Indeed, gene set testing revealed that genes encoding for the proteins overrepresented in the MEx-treated group were overexpressed in nonclassical 
A



Day 7
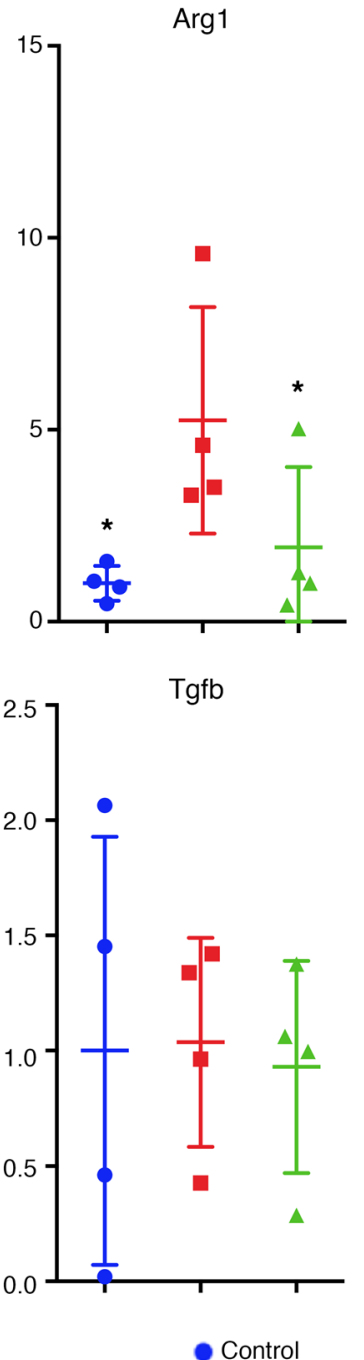

B
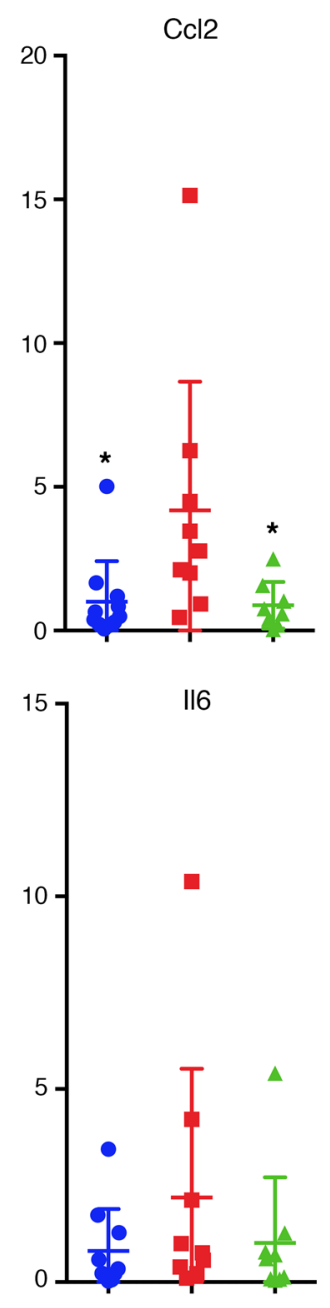

Day 14
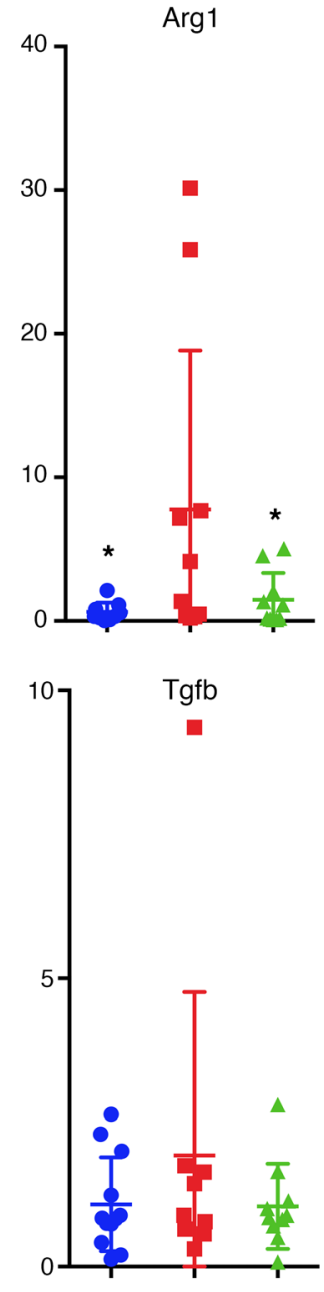

Bleomycin $\quad \Delta$ Bleo + MEx

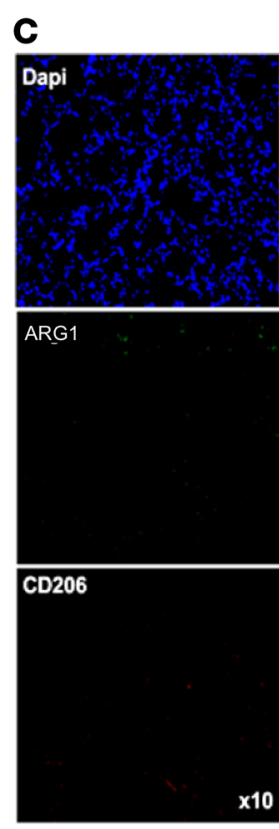

Control


Bleomycin
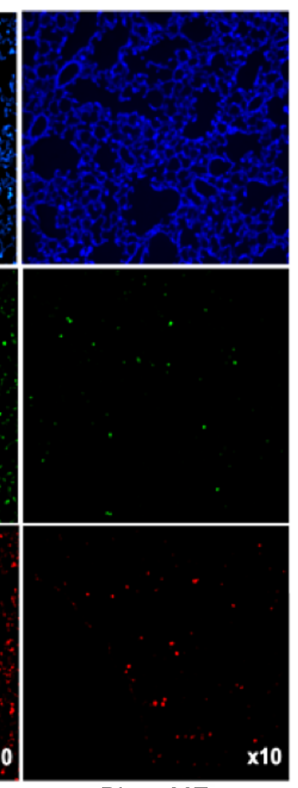

Bleo+MEx
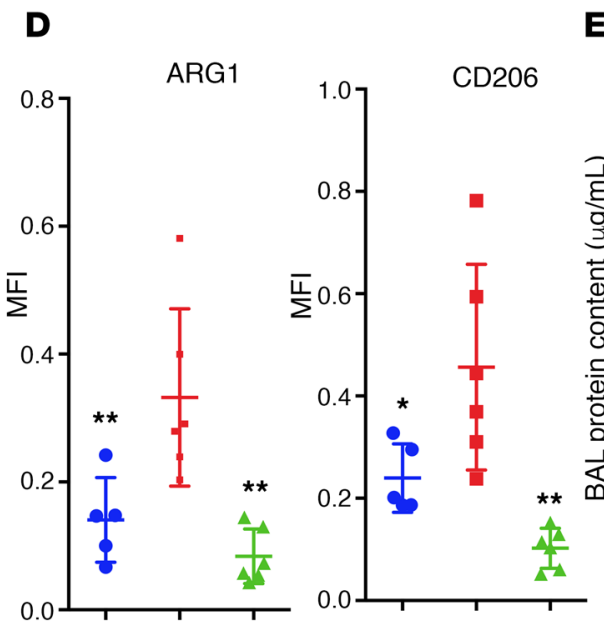

E

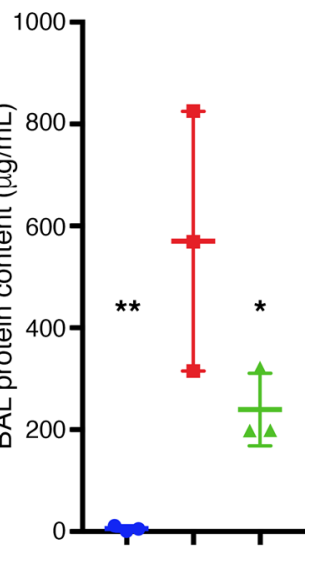

- Control

Bleomycin

$\triangle \mathrm{Bleo}+\mathrm{MEX}$ 
Figure 5. Administration of MEx modulates whole lung inflammation. (A and B) Whole lung qPCR demonstrated an increase in the mRNA expression levels of $C c / 2$ and Arginase1 (Arg1) at days 7 and 14 in bleomycin-challenged animals compared with untreated control mice. This was ameliorated by MEx treatment. (C and D) $/ 16$ expression showed a similar trend, but statistical significance was not achieved. Levels of Tgfb remained unchanged between the groups. Results are expressed relative to control expression (fold change). Immunofluorescence analysis of lung sections using antibodies against ARG1 (green) and CD206 (red) . Nuclei staining performed with DAPI (blue). Images obtained at $\times 10$ magnification. Mean fluorescence intensity (MFI) normalized for cell number (DAPI stain). Analysis performed by Imagel software. Mean \pm SD, $n=5$-8 per group; each symbol represents 1 mouse. ${ }^{*} P<0.05$; ${ }^{* *} P$ $<0.01$. One-way ANOVA followed by Fisher's LSD post hoc analysis. (E) BAL protein content was decreased after MEx treatment. Mean \pm SD, $n=3-4$ per group; each symbol represents 1 mouse. ${ }^{*} P<0.05$; ${ }^{*} P<0.01$. One-way ANOVA followed by Fisher's LSD post hoc analysis. BAL, bronchoalveolar lavage.

Ly6 $\mathrm{c}^{\text {neg }}$ monocytes $(P<0.001)$, whereas genes encoding for the proteins under-represented in MEx-treated My/Mo cells were overexpressed in classical Ly6c ${ }^{\text {pos }}$ monocytes $(P<0.001)$ (Figure 7D and Supplemental Figure 2). These results provide compelling evidence that the protective effects induced by $\mathrm{MEx}$ treatment are, at least in part, mediated by reprogramming of BMDMo to a nonclassical phenotype.

Transplantation of BMDMo preconditioned with MEx prevents bleomycin-induced pulmonary fibrosis. Since our proteomic data indicated a primary effect of MEx on monocyte phenotypes within the BMDMy populations, we sought to address whether the promodulatory effect of MEx on BMDMo is responsible for the prevention of fibrosis. We performed adoptive transfer experiments of BMDMo that were preconditioned with MEx (as shown in the schematic of Figure 8A) ex vivo and delivered into the bleomycin-induced pulmonary fibrosis model. Mice that received 2 doses of BMDMo on days 0 and 3 after bleomycin injection were sacrificed at day 14 and lungs were assessed for histology and collagen content (Figure 8). Compared with bleomycin control mice, the group that received BMDMo preconditioned with MEx (BMDMo+MEx) presented with a drastically improved Ashcroft score (7.2 \pm 0.3 vs. $3.5 \pm 1, P<0.0001$, respectively) coupled with lower total collagen levels $(3.02 \pm 0.23$ vs. $0.68 \pm 0.41 \mathrm{mg} / \mathrm{mL}, P<0.001$, respectively). Interestingly, mice that received BMDMo control (not preconditioned with MEx, [BMDMo+Media]), presented with evidence of partially reduced fibrosis, as assessed by the Ashcroft score ( $P$ $<0.05$ ), however collagen deposition was similar between the 2 experimental groups (Figure 8, D and E).

To explore if the antifibrotic effect of MEx is due to resident alveolar $M \Phi$, we also administered alveolar MФ preconditioned with MEx (alveolar MФ+MEx) endotracheally following bleomycin instillation. Again, alveolar M $\Phi$ were sourced from bronchoalveolar lavage fluid (BALF) obtained from WT (healthy) FVB mice aged 6-8 weeks. Importantly, we did not detect any amelioration of fibrosis in mice who received preconditioned alveolar MФ compared with the bleomycin group (Figure 8, C, D, and E). To further assess the inflammatory changes in the lung after the administration of MEx-preconditioned BMDMo, we quantified the Cd68- and Retnla-expressing MФ in the pulmonary parenchyma using immunofluorescent staining. Mice that received monocytes and were preconditioned with MEx showed reduced pulmonary МФ numbers compared with BMDMo plus Media-treated littermates $(P<0.05$, Figure 9$)$.

\section{Discussion}

Here, we demonstrate that a single i.v. dose of purified exosomes derived from human BM MSCs effectively prevented and reverted core features of bleomycin-induced pulmonary fibrosis, improving pulmonary morphology, blunting collagen deposition, and restoring lung architecture. Furthermore, we show that MEx treatment is associated with an analogous modulation of lung and BM monocyte populations. We demonstrated a shift in the proportion of infiltrating classical monocytes, nonclassical monocytes, and alveolar $\mathrm{M} \Phi$ to favor the monocyte/M $\Phi$ profiles of untreated control mice. We further extended our observations to show that the systemic modulatory role and prohomeostatic nonclassical effect of MEx is primarily executed by the modulation of myeloid cell phenotype. Proteomic analysis of MEx-preconditioned monocyte revealed a signature indicative of proregulatory (Ly6 $\mathrm{c}^{\mathrm{lo}}$, nonclassical) monocytes. Strikingly, we found that transplantation of BMDMo that were preconditioned by MEx prevented collagen deposition, restored lung architecture in bleomycin-exposed animals, and decreased inflammation.

Previous reports established that MSC/stem cell-based therapies have been effective in preventing experimental models of pulmonary fibrosis $(11,13)$. Although the findings from this report should be judged in the context of the bleomycin experimental model used, our findings here are in accordance with our previous reports, which demonstrate that the MSC secretome harnesses the prominent therapeutic modalities and that the major therapeutic vector within the conditioned media is represented by the exosomes ( 15 , 18). The potent immunomodulatory role of MEx and MSC conditioned media has been well recognized in preclinical models of bronchopulmonary dysplasia, pulmonary hypertension, cardiotoxin-induced skeletal 
A

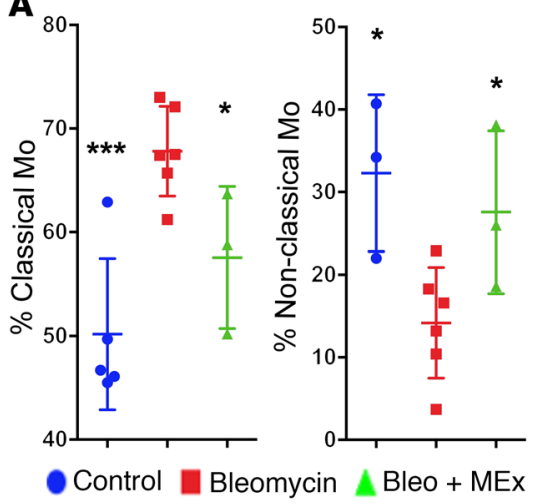

B

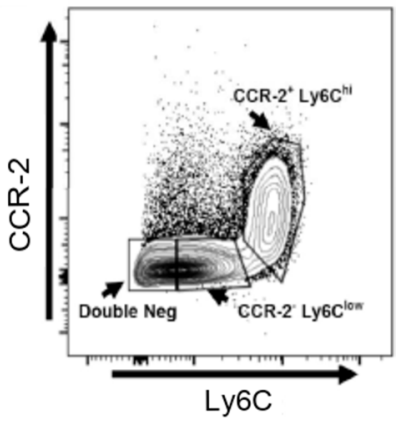

Figure 6. MEx modulate monocyte phenotype in the BM. (A) To investigate the systemic effects of MEx, we analyzed the myeloid cell profile of the BM at day 7 by flow cytometry. Classical monocytes (Classical Mo) were defined as CD45+CD11b+MHCII-CD64-CCR-2+Ly6C hi. Nonclassical monocytes (Nonclassical Mo) were defined as CD45+CD11b+MHCII-CD64-CCR-2-Ly6C ${ }^{\circ}$. (B) Representative gating strategy. Data represents mean \pm SD, $n=5$-8 per group; each symbol represents 1 mouse ${ }^{*} P<0.05 ;{ }^{* *} P<0.001$. One-way ANOVA followed by Fisher's LSD post hoc analysis.

muscle injury, and acute lung injury $(19,30,31)$. In agreement, our present study not only suggests that MEx therapy modulates whole lung inflammation but demonstrates a potentially novel immunomodulatory effect of MEx on lung and BM monocyte populations. Specifically, we report that MEx therapy initiates an increase in nonclassical monocytes and a concurrent reduction in proinflammatory monocytes in the BM. This is associated with similar immunomodulatory effects in the lung and with drastic improvements in lung architecture and the subsequent prevention of bleomycin-induced pulmonary fibrosis. Interestingly, MEx-preconditioned monocytes exhibit a proteomic signature compatible with nonclassical monocytes.

In experimental models of fibrosis, it is well recognized that recruitment of inflammatory (Ly6C ${ }^{\text {hi }}$ ) monocytes and chronic activation of proremodeling $\mathrm{M} \Phi$ play an important role in the development and progression of fibrosis (24-26, 30, 32-36). For example, using a diphtheria toxin receptor-induced acute kidney injury model, Lin and colleagues have shown that BM Ly6 $\mathrm{C}^{\text {hi }}$ monocytes are selectively recruited to injured kidney and play a pivotal role in regulating fibrosis (36). Previous reports have also demonstrated that patients with IPF present with differential expression of monocyte/MФ-specific markers (37) and that altered monocytic profiles may contribute to the pathogenesis of interstitial lung diseases (ILD) (38). More recent studies investigating the transcriptome profile of monocyte/MФ populations in a bleomycin model of pulmonary fibrosis, suggest that selectively targeting monocyte-derived alveolar M $\Phi$ differentiation may ameliorate pulmonary fibrosis (39).

The striking prevention of bleomycin-induced pulmonary fibrosis from transplantation of MEx-preconditioned BMDMo implies that the systemic modulation of BMDMo phenotype is likely associated with the antifibrotic actions of MEx in the lung. Notably, no beneficial effects were found with transplantation of MEx-preconditioned alveolar MФ. In contrast, using a LPS-induced acute lung injury model, Morrison and colleagues recently demonstrated that endotracheal administration of alveolar MФ preconditioned with MSC EVs decreased whole lung inflammation and BALF protein (30). It is fair to speculate that this may be due to the differences in experimental models and subsequent underlying pathophysiology due to distinct $М \Phi$ phenotypes and mechanism of injury. van de Laar et al. demonstrated that both mature alveolar MФ and BMDMo have the capacity to colonize an empty alveolar MФ niche and develop into functional tissue-resident $\mathrm{M} \Phi$ (40). Indeed, it is possible that the absence of an empty alveolar $M \Phi-$-specific niche at the beginning of inflammation (day 0-3) in our model did not allow sufficient colonization by the transplanted alveolar МФ. Furthermore, given our findings that "normal" BM contains a fraction $(\sim 32 \%)$ of nonclassical monocytes, it is expected that the adoptive transfer of monocytes that have not been exposed to MEx may confer some benefit. However, this beneficial effect is much reduced when compared with MEx-preconditioned BMDMo. In agreement, we show that MEx therapy not only ameliorates total lung dysregulation of MФ phenotype, but it is also associated with an analogous immunomodulatory effect on both the lung and BM myeloid cell populations. 
A

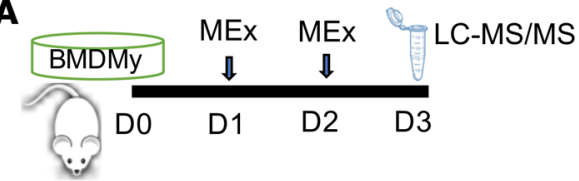

B
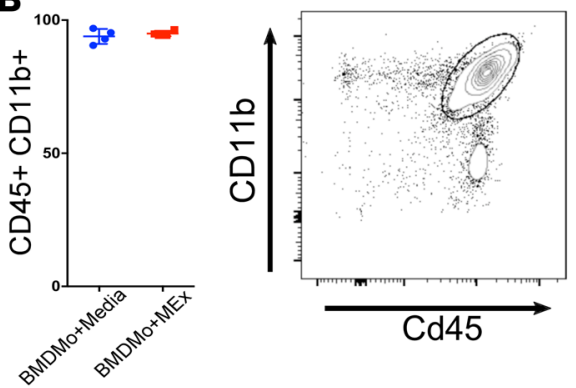

D

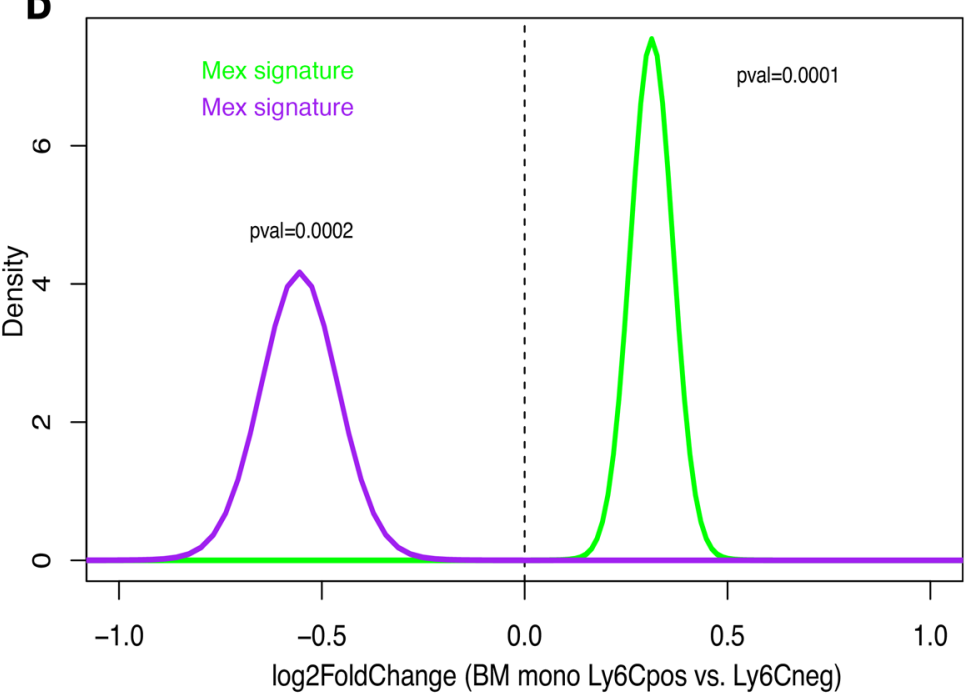

C

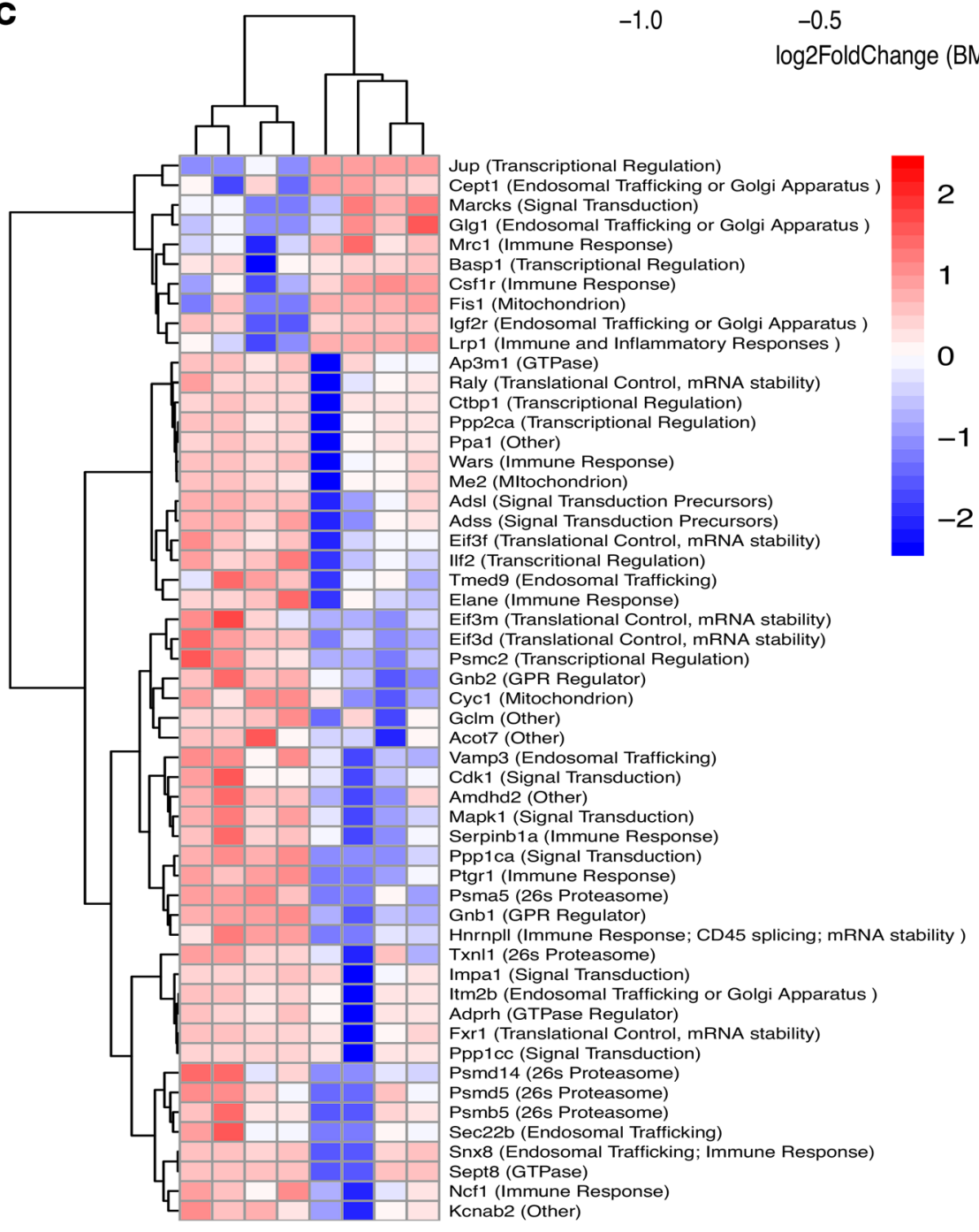

Figure 7. MEx therapy reprograms monocytes to a nonclassical (Ly6C $C^{\text {neg }}$ ) phenotype. (A) BM-derived myeloid cells (BMDMy) were isolated from 6- to 8-week-old FVB mice, cultured ex vivo for 3 days to drive the monocytic lineage, and treated with MEx (MEx dose: $1 \times 10^{6}$ MSC equivalents) or media alone on day 1 (D1) and day 2 (D2). (B) Flow cytometric analysis of BM-derived monocytes (BMDMo) after 3 days of culture showed that $>90 \%$ were CD45+CD11b+ cells. Student's $t$ test ( 2 tailed). To assess the direct effect of MEx on monocyte phenotype, BMDMo were treated with 2 doses of MEx or FEx on days 1 and 2 , and cells were processed for liquid chromatography-tandem mass spectrometry (LC-MS/MS) on day 3, as shown in A. (C) Eighty-four peptides where deemed differentially abundant in MEx-treated BMDMo vs. FEx-treated controls at FDR $<0.25$. (D) Gene set analysis revealed that genes encoding for peptides abundant in MEx-treated BMDMo were, on average, overexpressed in the Ly6C ${ }^{\text {neg }}$ monocytes $(P<0.001)$, whereas genes encoding for peptides abundant in the FEx-treated BMDMo were, on average, overexpressed in the Ly6 ${ }^{\text {pos }}$ monocytes $(P<0.001)$. Transcriptome data were obtained from GSE95411.

FEx-treated MEx-treated

Bioinformatics analysis identified 84 proteins to be differentially abundant in MEx- vs. FEx-preconditioned monocytes. This protein signature highlighted a decrease in proinflammatory state and an increase in proregulatory state of monocytes in response to MEx treatment. We could further establish an association between the MEx-induced proteomic signature and Ly6 $\mathrm{C}^{\text {neg }}$ proregulatory monocytes at the transcriptome level. Collectively, these analyses reinforce the hypothesis that MEx act directly on regulatory 
A

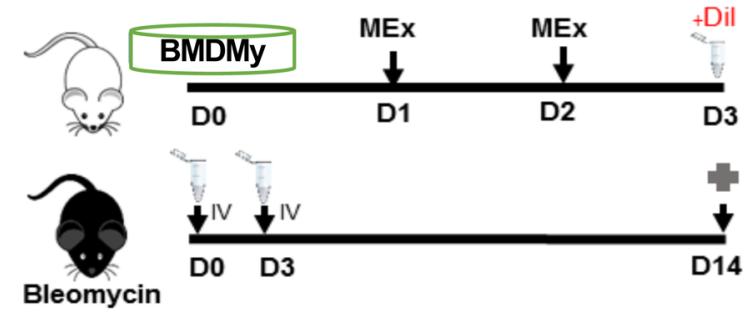

B

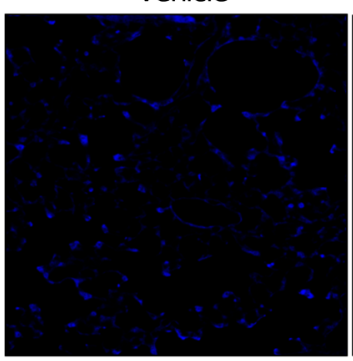

D

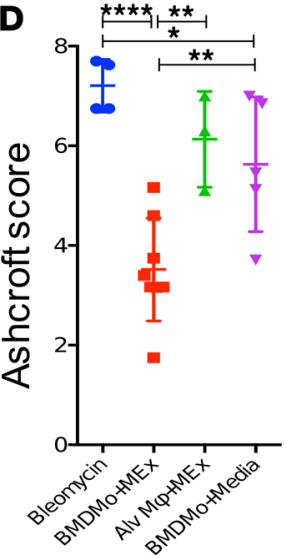

BMDMo

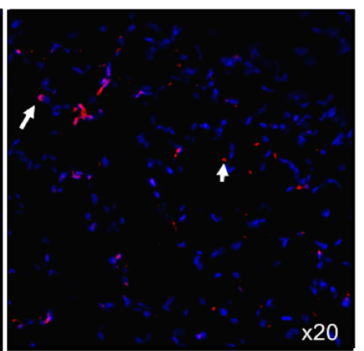

E

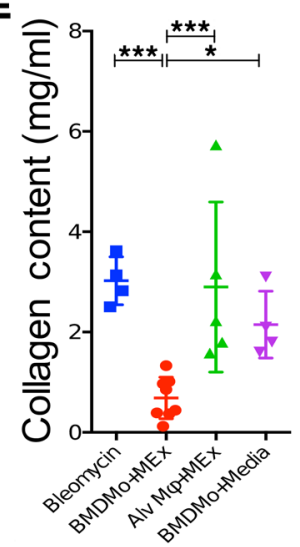

C

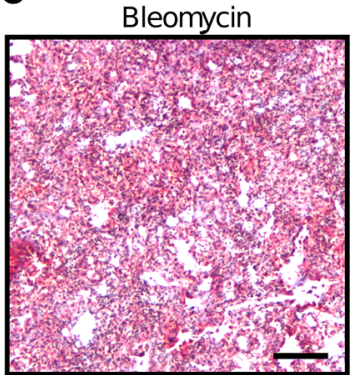

Alveolar MФ+MEx

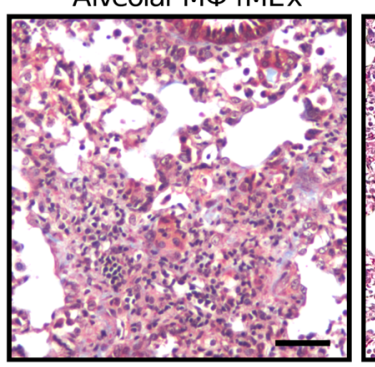

BMDMo+MEx

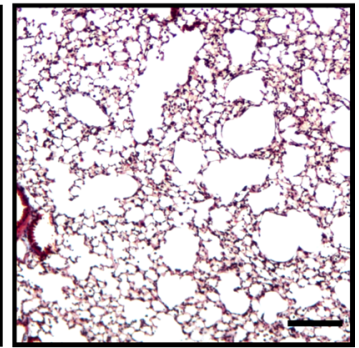

BMDMo+Media

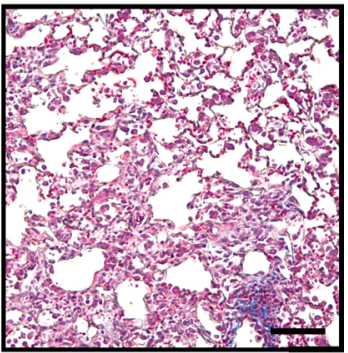

Figure 8. Transplantation of BM-derived monocytes preconditioned with MEX prevents bleomycin-induced pulmonary fibrosis. (A) We explored the effects of ex vivo treated BM-derived monocytes and alveolar macrophages (alveolar M $\Phi$ ) in the prevention of fibrosis. BMDMo were stained with lipophilic dye (Dil) on day 3 (D3) and i.v. administered at a 1:1 ratio on days 0 and 3 to C57BL/6 mice following endotracheal instillation of bleomycin. Mice were sacrificed at day 14. Cross symbol represents animal assessment. (B) Dil-labeled BMDMo were detected in the lung 14 days after injection, while no signal was seen in the lung of mice that received cell-free dye (vehicle). Images obtained at $\times 20$ magnification. Arrow marks the Dil-labeled monocytes. (C-E) Pulmonary fibrosis was ameliorated in mice that received monocytes that were preconditioned with $M E x$, while alveolar M $\Phi$ had little effect. Inserts were taken at $\times 100$ magnification. Data are representative of at least 2 independent experiments. Mean \pm SD, $n=4-5$ per experimental group; each symbol represents 1 mouse. Between-group comparison: ${ }^{*} P<0.05$; ${ }^{* *} P<0.01 ;{ }^{* *} P<0.001 ;{ }^{* * *} P<0.0001$. One-way ANOVA followed by Fisher's LSD post hoc analysis. Scale bar: $100 \mu \mathrm{m}$. Dil, 1,1'-Dioctadecyl-3,3,3',3'-Tetramethylindocarbocyanine Perchlorate ("Dil”; DilC18(3)).

pathways to reprogram monocytes into an antiinflammatory state. Further, findings from this report show that MEx, at least in part, via modulation of BM and lung monocyte/MФ profiles, suppress whole lung inflammation to favor prohomeostatic myeloid population phenotypes, which is associated with the prevention and reversal of bleomycin-induced pulmonary fibrosis.

Deciphering the molecular mechanism of action and identifying any specific bioactive modalities responsible for the beneficial effects of MEx remain pending. We strongly believe that this beneficial effect is not exerted via one mediator, but the constellation of mediators such as proteins and noncoding RNAs packaged in exosomes (41).

We noted the most remarkable results with early administration of MEx (day 0 and 7). Despite no improvement in Ashcroft score, we noted a significant reduction of collagen deposition with late administration of MEx (day 21). In the absence of inflammation in established fibrotic stage, we believe that other mechanisms such as nucleic acid transfer (41), inhibition of alveolar epithelial cell apoptosis (42), or promotion of endogenous growth factor secretion (43) may take part in the antifibrotic effect of MEx. 
A

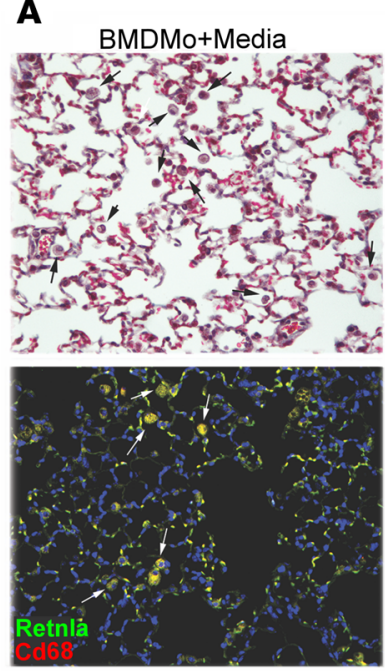

BMDMo+MEx

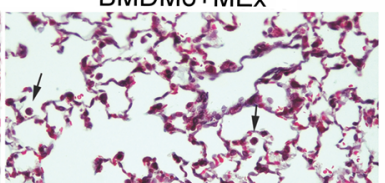

B

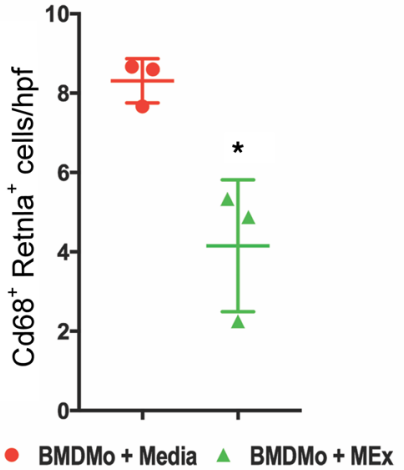

Figure 9. Transplantation of BM-derived monocytes preconditioned with MEx prevents bleomycin-induced pulmonary inflammation. (A and B) Pulmonary inflammation was analyzed at day 14 after the injection of MEx-preconditioned BMDMo (see details in Figure 8) by counting Cd68- and Retnla-expressing macrophages in the pulmonary parenchyma. Mice that received monocytes that were preconditioned with MEx showed reduced macrophage numbers compared with BMDMo+media-treated littermates. Images were taken at $\times 200$ magnification. Arrows mark inflammatory macrophages in alveolar spaces. Data are representative of at least 2 independent experiments, $n=3-5$ per experimental group. Mean \pm SD; each symbol represents 1 mouse. Between-group comparison: ${ }^{*} P<0.05$, Student's $t$ test (2-tailed). Scale bar: $100 \mu \mathrm{m}$.

Future studies beyond the scope of this manuscript should investigate how MEx may alter the epigenetic landscape and regulatory gene expression of BMDMo. Additionally, the effect of MEx on other cell types in IPF such as lung epithelial cells and endothelial cells needs further investigation.

On balance, the application of exosomes represents an exciting and innovative approach to treat fibrotic lung diseases, especially considering that exosome-based therapeutics may obviate safety concerns associated with live cell treatments. However, we acknowledge a number of limitations in our study. Firstly, MEx dose was based on previous work using MEx in experimental models of bronchopulmonary dysplasia and pulmonary hypertension. However, for considering the clinical application of MEx, dose response experiments should be performed in future studies. Additionally, future experiments beyond the scope of this study could investigate different routes of administration, specifically comparing endotracheal administration vs. i.v., to decipher if localized delivery is sufficient to afford systemic immunomodulatory benefits and to ameliorate bleomycin-induced pulmonary fibrosis. It is worth mentioning that, although our isolation method mostly allows for the purification of EV in the exosomal range, due to overlap of exosomes with microvesicles in size and morphology, the presence of microvesicles in our preparation is very likely.

Our group and others have shown that MSC exosomes distribute primarily to the lung, liver, and spleen $(44,45)$. It is plausible that MEx exert their upstream immunomodulatory effect in the BM by reprogramming the myeloid cells to a proregulatory phenotype, leading to lower lung infiltration of proinflammatory/profibrotic monocytes. An effective tool to track MEx in vivo and accurately assess the biodistribution remains elusive, and it is an important question to address for potential monocyte/MФ-targeted therapies.

On balance, findings described in this report provide insights into the systemic immunomodulatory responses following bleomycin-induced lung injury and the subsequent nonclassical effects of MEx-modulated monocyte phenotypes. Collectively, MEx treatment represents a promising cell-free therapy for the treatment of fibrotic lung diseases.

\section{Methods}

Exosome isolation and purification. Exosome isolation, purification, and characterization were performed as previously described using iodixanol (OptiPrep) cushion density flotation (19). Briefly, concentrated conditioned media from human BM MSCs or HDFs were floated on an iodixanol cushion and centrifuged for 3.5 hours at $100,000 \mathrm{~g}$ at $4^{\circ} \mathrm{C}$. The exosome-containing fraction (F9) was used for subsequent in vitro and in vivo experiments 
after confirming the presence of established exosome markers (ALIX, CD63, CD9, and FLOT1) (19).

Bleomycin-induced pulmonary fibrosis model. Fourteen-week-old mice (C57BL/6 strain, Charles River Laboratories) were anesthetized with isoflurane and received a single endotracheal dose of bleomycin sulphate $(50 \mu \mathrm{L}, 3 \mathrm{U} / \mathrm{kg}$ ) at day 0 . Bleomycin naive mice (control) received an endotracheal dose of saline (50 $\mu \mathrm{L})$. Treated animals received a single i.v. (tail vein) dose of $\mathrm{MEx}\left(200 \mu \mathrm{L}\right.$; dose, $5 \times 10^{6} \mathrm{MSC}$ equivalents; $\sim 8.6 \times 10^{8}$ particles) at day 0 . HDF-derived exosomes (FEx; $200 \mu \mathrm{L}$; dose, $5 \times 10^{6} \mathrm{HDF}$ equivalents; $\sim 9.2 \times$ $10^{8}$ particles) or exosome-free iodixanol vehicle only $(200 \mu \mathrm{L} ; 10 \% \mathrm{w} / \mathrm{v}$; iodixanol [IDX]) served as biological and vehicle controls, respectively. Mice were assessed at day 7 and/or at day 14 or day 28 for cytometric, histological, and/or quantitative PCR (qPCR) analysis.

Cell isolation and culture. Human BM mesenchymal stem cells were obtained from RoosterBio. HDFs were established by tissue explant method (46). BM MSCs and HDFs were cultured, expanded, and further characterized as described previously (19).

TEM. An aliquot of 5-10 $\mu \mathrm{L}$ of the exosome preparation was adsorbed for 15 seconds on a formvar/carbon-coated grid (Electron Microscopy Sciences). Samples were stained with 2\% uranyl acetate after removal of excess liquid with Whatman Grade 1 filter paper (MilliporeSigma). EVs were then viewed by a JEOL $1200 \mathrm{EX}$ transmission electron microscope (TEM), and images were recorded with an AMT 2k CCD camera.

NTA. Size and concentration distributions of exosomes were determined using NTA (NanoSight LM10 system, Malvern instruments) as described previously (19).

Western blot analysis. Proteins in exosome preparations were separated on a 4-20\% polyacrylamide gel (Bio-Rad), followed by transfer to $0.45 \mu \mathrm{m}$ PVDF membrane (MilliporeSigma). Rabbit polyclonal antiFLOT1 and anti-CD63 antibodies (Santa Cruz Biotechnology Inc.; 25506 and 15363, respectively), and mouse monoclonal anti-ALIX, anti-CD9, and anti-GM130 antibodies (Santa Cruz Biotechnology Inc.; 53538,13118 , and 55590, respectively) were used based on recommended dilutions by the manufacturer.

Exosome dosing. Exosome preparations were diluted on PBS to correspond to $5 \times 10^{6}$ cell equivalent. This dose was estimated based on our previous dose calculation in newborn mice with corresponding NTA and protein concentrations (19).

Histology. Mice were euthanized with i.p. injection of pentobarbital at designated time points following the instillation of bleomycin. The hearts were perfused with PBS (Invitrogen) through the right ventricle. BALF was collected after the instillation of $3 \mathrm{~mL}$ of intratracheal PBS (5 gentle collection of $0.6 \mathrm{~mL}$ ), cells were discarded after ultracentrifugation, and supernatant was stored for further analysis. BAL protein was quantified using Pierce BCA protein assay (Thermo Fisher Scientific). For histologic analysis, the trachea was cannulated, and lungs were inflated with $4 \%$ paraformaldehyde (PFA) at a constant pressure with a tubbing connected to a PFA reservoir $25 \mathrm{~cm}$ above the level of the mouse. The trachea was secured with a knot to avoid the drainage of PFA. The right lung was embedded in paraffin and sectioned for H\&E or Masson's trichrome staining. The left lung was either snap frozen in liquid nitrogen and used for RNA and protein isolation or used fresh for collagen quantification or cytometric analysis. Randomly selected areas (10-15 fields) from 5- $\mu \mathrm{m}$ thick lung sections were acquired at $\times 100$ and $\times 200$ magnification using a Nikon Eclipse 80i microscope (Nikon). For histologic quantification, the Ashcroft score was used in a blinded fashion. Scores of $0-1$ represented no fibrosis, scores of 2-3 represented minimal fibrosis, scores of 4-5 were considered as moderate fibrosis, and scores of 6-8 indicated severe fibrosis.

Immunofluorescence staining. Lung tissue sections were deparaffinized in xylene and rehydrated. Tissue slides were treated with $10 \mathrm{mM}$ citrate buffer and blocked with serum and BSA for 20 minutes. Samples were then incubated at $4^{\circ} \mathrm{C}$ overnight with indicated primary antibody Arg-1 (Santa Cruz Biotechnology Inc.; 20150), CD206 (Santa Cruz Biotechnology Inc.; 58987), CD68 (Bio-Rad; MCA1957GA), and Retnla (Abcam; 39626) and then further incubated with secondary antibody (Alex Fluor 488-conjugated goat anti-rabbit, A-11008; Alexa Fluor 594-conjugated rabbit anti-rat, A-21211) for 20 minutes, followed by nuclear staining with DAPI for 10 minutes. Arg1 ${ }^{+}$and $\mathrm{CD}^{2} 26^{+}$cells were imaged using a Nikon Eclipse 80i microscope. Ten random images were analyzed using ImageJ software (NIH). Mean Fluorescence Intensity (MFI) was calculated using the following formula: MFI = integrated density - (area of selected cell $\times$ mean fluorescence of background reading).

Sircol collagen assay. The left lung was used for collagen quantification per manufacturer's protocol (Bicolor, Life Science Assays). Briefly, left lung homogenate was shaken overnight at $4^{\circ} \mathrm{C}$ in $5 \mathrm{~mL}$ of 0.5 $\mathrm{M}$ acetic acid with $0.6 \% \mathrm{v} / \mathrm{v}$ pepsin. A total of $1 \mathrm{~mL}$ of dye reagent was added to $100 \mu \mathrm{L}$ of transparent supernatant, and the samples were vortexed for 30 minutes. The residual pellet was washed by acid-salt 
wash buffer to eliminate unbound collagen, and $\mathrm{pH}$ was normalized with alkalization buffer. Absorbance was measured at a wavelength of $550 \mathrm{~nm}$ in a microplate reader. Measured collagen content was compared with a standard curve and represented as $\mathrm{mg} / \mathrm{mL}$ of left lung homogenate.

Cytometric analysis of mouse whole lung and BM. Lung MФ populations were assessed by flow cytometry as previously described (47). Lungs were harvested on days 7 and 14. Left lung was cut into small pieces and digested in $5 \mathrm{~mL}$ of digestion buffer consisting of RPMI-1640 (Invitrogen), collagenase IV (1.6 $\mathrm{mg} / \mathrm{mL}$ ), and DNAse1 (50 unit/mL), (both from Worthington Biochemical Corp.). Lung were shaken at $37^{\circ} \mathrm{C}$ for 30 minutes, and RBC were lysed using RBC lysis buffer (Roche). Homogenized lung was passed through a $40-\mu \mathrm{m}$ cell strainer (Corning) to obtain a single-cell suspension.

For the assessment of alveolar M $\Phi$ and monocyte populations, the cell suspension was stained with antibodies PE/Cy7-conjugated anti-mouse CD45 (BioLegend; 103114), FITC-conjugated anti-mouse CD11b (BD Biosciences; 553310), PerCP Cy 5.5-conjugated anti--mouse CD11c (BD Pharmingen; 560584), BV 605-conjugated anti-mouse MHC II (BioLegend; 107639), PE-conjugated anti-mouse CD64 (BioLegend; 139303), BV 421-conjugated anti-mouse CD24 (BioLegend; 101825), BV 510-conjugated anti-mouse Ly6C (BioLegend; 127627), and Alexa 647-conjugated anti-mouse CCR-2 (BioLegend; 150603).

For the evaluation of BMDMo, freshly flushed cells from the femur and tibia of adult mice were stained with PE/Cy7-conjugated anti-mouse CD45, FITC-conjugated anti-mouse CD11b, BV 605-conjugated anti-mouse MHC II, PE-conjugated anti-mouse CD64, BV 421-conjugated anti-mouse CD24, BV 510-conjugated anti-mouse Ly6C, and Alexa 647-conjugated anti-mouse CCR-2.

Compensation was adjusted accordingly and supported by UltraComp ebeads (Affymetrix). Fluorescence-minus-one controls were used accordingly. Cell populations were identified according to the gating strategy illustrated in Supplemental Figure 2 and recorded as a percentage of total cell population.

Reverse transcription PCR analysis. Total RNA was extracted from left lung using TRIZOL (Thermo Fisher Scientific) as per manufacturer's instructions. Taqman primers used in the PCR reactions, including Ccl2, I16, Tgfb, and Arg-1, were obtained from Invitrogen. Nuclear pore protein 133 served as an internal control.

Annexin V/PI apoptosis assay, TUNEL staining. Annexin V staining kit (Sigma-Aldrich) was used to assess apoptosis in the whole lung. Single cell suspension was obtained from left lung as described above. Cells were then floated in $1 \times$ binding buffer and stained with FITC-conjugated annexin $\mathrm{V}$ and propidium iodide (PI) antibody for 10 minutes and immediately assessed by flow cytometry.

Apoptosis was assessed in paraffin-embedded lung tissue using TACS TdT in situ - Fluorescein TUNEL assay (R\&D Systems) per manufacturer protocol. Briefly, deparaffinized lung sections were permeabilized using Cytonin for 1 hour and labeled with a combination of Manganese cation, TdT dNTP Mix, and TdT enzyme (R\&D Systems), followed by incubation with Strep-Fluor solution for 20 minutes. Fluorescent imaging and quantification were performed as described above.

Adoptive transfer of MEx treated BMDMo. BMDMy were isolated from 6- to 8-week-old FVB by flushing the femur and tibia and culturing cells for 3 days in DMEM (Sigma-Aldrich) supplemented with $10 \% \mathrm{FBS}$, containing 30\% v/v L929 conditioned medium (as a source of MФ CSF; M-CSF). Each well was treated with MEx generated from $1 \times 10^{6}$ MSCs or media only on days 1 and 2. Cells were harvested on day 3 and after 2 washes with PBS and stained with DiI (Thermo Fisher Scientific) as per the manufacturer's protocol (Invitrogen). BMDMo were then administered via tail vein injection at a 1:1 ratio (BMDMo isolated from 1 mouse were injected into the experiment mouse; approximately $3 \times 10^{6}$ cells) on day 0 and day 3 after endotracheal instillation of bleomycin.

Adoptive transfer of MEx-treated murine-derived alveolar MФ. Six- to 8-week-old FVB mice were euthanized by i.p. pentobarbital injection. The anterior wall of the trachea was cannulated with a 21-gauge needle and secured using a string. BALF was collected with 5 flushes of $0.6 \mathrm{~mL}$ of sterile HBSS (supplemented with $0.5 \mathrm{mM}$ EDTA and $1 \mathrm{mM}$ HEPES) using a 1-mL syringe. BALF was centrifuged at $400 \mathrm{~g}$ for 5 minutes, and the supernatant was aspirated. Murine alveolar MФ were resuspended in fresh RPMI media (Sigma-Aldrich) supplemented with 1\% penicillin/streptomycin and $10 \% \mathrm{FBS}$ and were seeded in a $35-\mathrm{mm}$ plate at a seeding density of $2 \times 10^{5}$ per plate. Each plate was treated overnight with MEx generated from $2 \times 10^{5}$ cells. The cells were harvested after 24 hours, washed twice with PBS, stained with DiI, and resuspended in $50 \mu \mathrm{L}$ of PBS. Alveolar MФ were administered endotracheally at a 1:1 ratio (alveolar MФ isolated from 1 mouse were administered to the experiment mouse; approximately $6 \times 10^{5}$ ) on day 0 and 3 following instillation of bleomycin.

Liquid chromatography-tandem mass spectrometry (LC-MS/MS). BMDMo were isolated from WT mice as previously described and treated with MEx (generated from $1 \times 10^{6} \mathrm{MSCs}$ ) or FEx (exosomes generated 
from $1 \times 10^{6}$ fibroblasts) on day 1 and day 2 . Cells were harvested on day 3 , and proteins were extracted using the administration of $0.1 \%$ SDS buffer.

For LC-MS/MS, a previously published procedure was used (48). Briefly, peptides were desalted using StageTips, dried in a vacuum concentrator, and separated by reverse phase chromatography using a Dionex ultimate 3000 RSLC nano UPLC system connected in-line with an Orbitrap Elite (Thermo Fisher Scientific). A database search was performed using Mascot 2.5 (Matrix Science) and SEQUEST in Proteome Discoverer v.1.4. against a murine Uniprot protein database. Data were further processed and inspected in Scaffold 4.8.4 (Proteome Software); quantitative values normalized to total spectra were extracted for downstream analysis.

Bioinformatics analysis. To compare the proteomic profiles of MEx- and FEx-preconditioned BMDMo $(n=$ 4 in each group), lowly abundant peptides were filtered out by keeping features with at least 10 counts in at least 4 samples irrespective of group labels. Following log transformation and quantile normalization, differentially abundant features were identified using a moderated t-statistic as implemented in the limma package from Bioconductor (49). Using an FDR threshold of 0.25 , we identified 84 peptides to be differentially abundant between MEx- and FEx-preconditioned BMDMo, hereafter referred to as the MEx-FEx proteomic signature.

To assess the enrichment of classical and nonclassical monocyte genes among the genes encoding for the MEx-FEx proteomic signature, we used a previously published transcriptome data of BM-derived Ly6 $\mathrm{C}^{\mathrm{pos}}$ and Ly6C $C^{\text {neg }}$ monocytes (29). In brief, raw counts and metadata were obtained from DEE2 database (50). Count data was normalized using the rlog variance stabilization functionality from DESeq2 Bioconductor package (51). Gene annotation was obtained using biomaRt package also from Bioconductor (52), and normalized count data was further summarized at the gene symbol level using the collapseRows functionality from WGCNA R package (53). In the case of more than 1 ENSEMBL gene ID mapping to the same gene symbol, normalized counts from the ENSEMBL ID with maximum variance were retained. Finally, self-contained gene set testing was performed using the QuSAGE package from Bioconductor (54). In short, QuSAGE summarizes differential expression of a given gene set by convoluting individual gene t-distributions into a single PDF for the gene set and tests the null hypothesis that the mean fold change is zero for a comparison of interest. All bioinformatics analysis were performed using the $\mathrm{R}$ statistical computing language (55).

Statistics. Data between 2 groups were compared using 2-tailed Student's $t$ test, and data between multiple experimental groups were compared using 1-way ANOVA with Fisher's LSD post hoc analysis in GraphPad Prism (v6.0). Flow cytometry data analyses were performed using FlowJo software v10.2 (Tree Star Inc.). Whole lung mRNA levels were assessed by qPCR relative to endogenous control (Nuclear pore protein 133 [Nup133]), where the $\Delta \mathrm{CT}$ was used for statistical analysis. Data are presented as mean $\pm \mathrm{SD}$. Significance was determined with respect to the $P<0.05$ threshold. For in vivo studies, sample size calculations were based on preliminary data (not shown), suggesting that detection of a $20 \%$ improvement in lung collagen content with a greater than $90 \%$ power at the $5 \% \alpha$-level would require a minimum of 5 animals per experimental group.

Study approval. Animal experiments were approved by the BCH Animal Care and Use Committee.

\section{Author contributions}

NM and GRW participated in study design and execution, data collection, analysis, and manuscript writing. AFG and MR participated in study design and execution, analysis, and manuscript writing. SN performed bioinformatics analyses and contributed to writing the manuscript. SAM and SK contributed to study design, supervision of study execution, analysis, manuscript writing, and final article editing and approval.

\section{Acknowledgments}

The authors thank Carolina Medrano and Roger Ilagan (United Therapeutics Corp., Durham, North Carolina, USA) for useful discussions. We also thank Xianlan Liu (Boston Children's Hospital, Boston, Massachusetts, USA) for expert animal husbandry, John Daley II and Susan Lazo (Dana Farber Cancer Institute, Boston, Massachusetts, USA) for their flow cytometry expertise and technical assistance, and Maria Ericsson for supporting electron microscopy experiments (Harvard Medical School, Boston, Massachusetts, USA). We thank Romain Hamelin at the EPFL proteomic facility for helping with sample preparation for mass spectrometry (EPFL, Lausanne, Switzerland). This work was supported in part by NIH grants R01HL146128 (SK); United Therapeutics Research Grant (SK and SAM); American Thoracic Society Foundation Grant (GRW); Little Giraffe Foundation (GRW); and Charles H. Hood Foundation Major Grants Initiative (SK). 
Address correspondence to: Stella Kourembanas, Boston Children's Hospital, 300 Longwood Avenue, Division of Newborn Medicine, Boston, Massachusetts 02115, USA. Phone: 617.919.2355; Email: stella.kourembanas@childrens.harvard.edu.

1. Gross TJ, Hunninghake GW. Idiopathic pulmonary fibrosis. N Engl J Med. 2001;345(7):517-525.

2. Brooks PJ, et al. Mortality after hip resurfacing versus total hip arthroplasty in young patients: a single surgeon experience. Ann Transl Med. 2019;7(4):77.

3. du Bois RM. Idiopathic pulmonary fibrosis: present understanding and future options. Eur Respir Rev. 2011;20(121):132-133.

4. Bingle L, et al. Differential epithelial expression of the putative innate immune molecule SPLUNC1 in cystic fibrosis. Respir Res. 2007;8:79.

5. Balestro E, et al. Immune Inflammation and Disease Progression in Idiopathic Pulmonary Fibrosis. PLoS ONE. 2016;11(5): $\mathrm{e} 0154516$.

6. Cheresh P, Kim SJ, Tulasiram S, Kamp DW. Oxidative stress and pulmonary fibrosis. Biochim Biophys Acta. 2013;1832(7):1028-1040

7. Kaunisto J, Salomaa ER, Hodgson U, Kaarteenaho R, Myllärniemi M. Idiopathic pulmonary fibrosis--a systematic review on methodology for the collection of epidemiological data. BMC Pulm Med. 2013;13:53

8. Aiuti A, et al. Gene therapy for immunodeficiency due to adenosine deaminase deficiency. N Engl J Med. 2009;360(5):447-458.

9. Harari S, Caminati A, Madotto F, Conti S, Cesana G. Epidemiology, survival, incidence and prevalence of idiopathic pulmonary fibrosis in the USA and Canada. Eur Respir J. 2017;49(1):1601504.

10. Toonkel RL, Hare JM, Matthay MA, Glassberg MK. Mesenchymal stem cells and idiopathic pulmonary fibrosis. Potential for clinical testing. Am J Respir Crit Care Med. 2013;188(2):133-140.

11. Ortiz LA, et al. Mesenchymal stem cell engraftment in lung is enhanced in response to bleomycin exposure and ameliorates its fibrotic effects. Proc Natl Acad Sci USA. 2003;100(14):8407-8411.

12. Ghadiri M, Young PM, Traini D. Cell-based therapies for the treatment of idiopathic pulmonary fibrosis (IPF) disease. Expert Opin Biol Ther. 2016;16(3):375-387.

13. Ortiz LA, et al. Interleukin 1 receptor antagonist mediates the antiinflammatory and antifibrotic effect of mesenchymal stem cells during lung injury. Proc Natl Acad Sci USA. 2007;104(26):11002-11007.

14. Srour N, Thébaud B. Mesenchymal Stromal Cells in Animal Bleomycin Pulmonary Fibrosis Models: A Systematic Review. Stem Cells Transl Med. 2015;4(12):1500-1510.

15. Lee C, et al. Exosomes mediate the cytoprotective action of mesenchymal stromal cells on hypoxia-induced pulmonary hypertension. Circulation. 2012;126(22):2601-2611.

16. Heldring N, Mäger I, Wood MJ, Le Blanc K, Andaloussi SE. Therapeutic Potential of Multipotent Mesenchymal Stromal Cells and Their Extracellular Vesicles. Hum Gene Ther. 2015;26(8):506-517.

17. Sdrimas K, Kourembanas S. MSC microvesicles for the treatment of lung disease: a new paradigm for cell-free therapy. Antioxid Redox Signal. 2014;21(13):1905-1915.

18. Willis GR, Kourembanas S, Mitsialis SA. Therapeutic Applications of Extracellular Vesicles: Perspectives from Newborn Medicine. Methods Mol Biol. 2017;1660:409-432.

19. Willis GR, et al. Mesenchymal Stromal Cell Exosomes Ameliorate Experimental Bronchopulmonary Dysplasia and Restore Lung Function through Macrophage Immunomodulation. Am J Respir Crit Care Med. 2018;197(1):104-116.

20. Cruz FF, et al. Systemic Administration of Human Bone Marrow-Derived Mesenchymal Stromal Cell Extracellular Vesicles Ameliorates Aspergillus Hyphal Extract-Induced Allergic Airway Inflammation in Immunocompetent Mice. Stem Cells Transl Med. 2015;4(11):1302-1316.

21. Moeller A, Ask K, Warburton D, Gauldie J, Kolb M. The bleomycin animal model: a useful tool to investigate treatment options for idiopathic pulmonary fibrosis? Int J Biochem Cell Biol. 2008;40(3):362-382.

22. Barbas-Filho JV, Ferreira MA, Sesso A, Kairalla RA, Carvalho CR, Capelozzi VL. Evidence of type II pneumocyte apoptosis in the pathogenesis of idiopathic pulmonary fibrosis (IFP)/usual interstitial pneumonia (UIP). J Clin Pathol. 2001;54(2):132-138

23. Hagimoto N, Kuwano K, Nomoto Y, Kunitake R, Hara N. Apoptosis and expression of Fas/Fas ligand mRNA in bleomycin-induced pulmonary fibrosis in mice. Am J Respir Cell Mol Biol. 1997;16(1):91-101.

24. Gibbons MA, et al. Ly6Chi monocytes direct alternatively activated profibrotic macrophage regulation of lung fibrosis. $A m J$ Respir Crit Care Med. 2011;184(5):569-581.

25. McCubbrey AL, et al. Deletion of c-FLIP from CD11 $\mathrm{b}^{\text {hi }}$ Macrophages Prevents Development of Bleomycin-induced Lung Fibrosis. Am J Respir Cell Mol Biol. 2018;58(1):66-78.

26. Misharin AV, et al. Monocyte-derived alveolar macrophages drive lung fibrosis and persist in the lung over the life span. $J$ Exp Med. 2017;214(8):2387-2404.

27. Satoh T, et al. Identification of an atypical monocyte and committed progenitor involved in fibrosis. Nature. 2017;541(7635):96-101.

28. Francke A, Herold J, Weinert S, Strasser RH, Braun-Dullaeus RC. Generation of mature murine monocytes from heterogeneous bone marrow and description of their properties. J Histochem Cytochem. 2011;59(9):813-825.

29. Mildner A, et al. Genomic Characterization of Murine Monocytes Reveals C/EBP $\beta$ Transcription Factor Dependence of Ly6C Cells. Immunity. 2017;46(5):849-862.e7.

30. Morrison TJ, et al. Mesenchymal Stromal Cells Modulate Macrophages in Clinically Relevant Lung Injury Models by Extracellular Vesicle Mitochondrial Transfer. Am J Respir Crit Care Med. 2017;196(10):1275-1286.

31. Hansmann G, et al. Mesenchymal stem cell-mediated reversal of bronchopulmonary dysplasia and associated pulmonary hypertension. Pulm Circ. 2012;2(2):170-181.

32. Bellón T, et al. Alternative activation of macrophages in human peritoneum: implications for peritoneal fibrosis. Nephrol Dial Transplant. 2011;26(9):2995-3005.

33. Braga TT, Agudelo JS, Camara NO. Macrophages During the Fibrotic Process: M2 as Friend and Foe. Front Immunol. 
$2015 ; 6: 602$

34. Kolahian S, Fernandez IE, Eickelberg O, Hartl D. Immune Mechanisms in Pulmonary Fibrosis. Am J Respir Cell Mol Biol. 2016;55(3):309-322

35. Mora AL, et al. Activation of alveolar macrophages via the alternative pathway in herpesvirus-induced lung fibrosis. $A m J$ Respir Cell Mol Biol. 2006;35(4):466-473.

36. Lin SL, Castaño AP, Nowlin BT, Lupher ML, Duffield JS. Bone marrow Ly6Chigh monocytes are selectively recruited to injured kidney and differentiate into functionally distinct populations. J Immunol. 2009;183(10):6733-6743.

37. Desai B, et al. Differential expression of monocyte/macrophage- selective markers in human idiopathic pulmonary fibrosis. Exp Lung Res. 2011;37(4):227-238.

38. Greiffo FR, Frankenberger M, Ortega-Gomez A, Behr J, Soehnlein O, Eickelberg O. Monocyte immunophenotyping reflects abberant activation patterns in interstitial lung disease patients. Am J Respir Crit Care Med. 2017;195:A6350.

39. Misharin AV, et al. Monocyte-derived alveolar macrophages drive lung fibrosis and persist in the lung over the life span. $J$ Exp Med. 2017;214(8):2387-2404.

40. van de Laar L, et al. Yolk Sac Macrophages, Fetal Liver, and Adult Monocytes Can Colonize an Empty Niche and Develop into Functional Tissue-Resident Macrophages. Immunity. 2016;44(4):755-768.

41. Ferguson SW, et al. The microRNA regulatory landscape of MSC-derived exosomes: a systems view. Sci Rep. 2018;8(1):1419.

42. Bernard O, et al. Mesenchymal stem cells reduce hypoxia-induced apoptosis in alveolar epithelial cells by modulating HIF and ROS hypoxic signaling. Am J Physiol Lung Cell Mol Physiol. 2018;314(3):L360-L371.

43. Dong LH, et al. The anti-fibrotic effects of mesenchymal stem cells on irradiated lungs via stimulating endogenous secretion of HGF and PGE2. Sci Rep. 2015;5:8713.

44. Wiklander OP, et al. Extracellular vesicle in vivo biodistribution is determined by cell source, route of administration and targeting. J Extracell Vesicles. 2015;4:26316.

45. Charoenviriyakul C, Takahashi Y, Morishita M, Matsumoto A, Nishikawa M, Takakura Y. Cell type-specific and common char acteristics of exosomes derived from mouse cell lines: Yield, physicochemical properties, and pharmacokinetics. Eur J Pharm Sci. 2017;96:316-322.

46. Nichols WW, Murphy DG, Cristofalo VJ, Toji LH, Greene AE, Dwight SA. Characterization of a new human diploid cell strain, IMR-90. Science. 1977;196(4285):60-63.

47. Misharin AV, Morales-Nebreda L, Mutlu GM, Budinger GR, Perlman H. Flow cytometric analysis of macrophages and dendritic cell subsets in the mouse lung. Am J Respir Cell Mol Biol. 2013;49(4):503-510.

48. Keklikoglou I, et al. Chemotherapy elicits pro-metastatic extracellular vesicles in breast cancer models. Nat Cell Biol. 2019;21(2):190-202.

49. Ritchie ME, et al. limma powers differential expression analyses for RNA-sequencing and microarray studies. Nucleic Acids Res. 2015;43(7):e47.

50. Ziemann M, Kaspi A, El-Osta A. Digital expression explorer 2: a repository of uniformly processed RNA sequencing data. Gigascience. 2019;8(4):giz022.

51. Love MI, Huber W, Anders S. Moderated estimation of fold change and dispersion for RNA-seq data with DESeq2. Genome Biol. 2014;15(12):550.

52. Durinck S, Spellman PT, Birney E, Huber W. Mapping identifiers for the integration of genomic datasets with the R/Bioconductor package biomaRt. Nat Protoc. 2009;4(8):1184-1191.

53. Langfelder P, Horvath S. WGCNA: an R package for weighted correlation network analysis. BMC Bioinformatics. 2008;9:559.

54. Yaari G, Bolen CR, Thakar J, Kleinstein SH. Quantitative set analysis for gene expression: a method to quantify gene set differential expression including gene-gene correlations. Nucleic Acids Res. 2013;41(18):e170.

55. R Core Team. R Foundation for Statistical Computing V, Austria. R: A language and environment for statistical computing. http://www.R-project.org. Accessed October 3, 2019. 